\title{
Second Chern Number and Non-Abelian Berry Phase in Topological Superconducting Systems
}

\author{
H. Weisbrich $\odot,{ }^{1, \dagger}$ R.L. Klees $\odot,{ }^{1, \dagger}$ G. Rastelli®, ${ }^{1,2,3}$ and W. Belzig ${ }^{1, *}$ \\ ${ }^{1}$ Fachbereich Physik, Universität Konstanz, D-78457 Konstanz, Germany \\ ${ }^{2}$ Zukunftskolleg, Universität Konstanz, D-78457 Konstanz, Germany \\ ${ }^{3}$ INO-CNR BEC Center and Dipartimento di Fisica, Universita di Trento, I-38123 Povo, Italy
}

(Received 9 July 2020; accepted 15 December 2020; published 19 January 2021)

\begin{abstract}
Topology ultimately unveils the roots of the perfect quantization observed in complex systems. The two-dimensional quantum Hall effect is the celebrated archetype. Remarkably, topology can manifest itself even in higher-dimensional spaces in which control parameters play the role of extra, synthetic dimensions. However, so far, a very limited number of implementations of higher-dimensional topological systems have been proposed, a notable example being the so-called four-dimensional quantum Hall effect. Here we show that mesoscopic superconducting systems can implement higher-dimensional topology and represent a formidable platform to study a quantum system with a purely nontrivial second Chern number. We demonstrate that the integrated absorption intensity in designed microwave spectroscopy is quantized and the integer is directly related to the second Chern number. Finally, we show that these systems also admit a non-Abelian Berry phase. Hence, they also realize an enlightening paradigm of topological non-Abelian systems in higher dimensions.
\end{abstract}

DOI: 10.1103/PRXQuantum.2.010310

\section{INTRODUCTION}

Topology ultimately explains the origin of phenomena that at first glance appear extremely fragile. The standard example is a gas of electrons confined in a plane under the effect of a strong magnetic field [the so-called twodimensional (2D) quantum Hall effect] [1,2], in which the Hall conductance is an integer in units of fundamental constants in physics, the elementary electron charge and the Planck constant. Real 2D electron systems are very far from being close to the simplified theoretical models and many microscopic details are inaccessible and unknown, such as structural disorder, atomic impurities, or edge configurations. Why simple toy models can explain such perfect quantization in real and complex systems remained mysterious until topology disclosed that all these systems are equivalent in the topological sense. Indeed, for the 2D quantum Hall effect, the quantized Hall conductance is related to the first Chern number of the occupied electronic bands [3].

*wolfgang.belzig@uni-konstanz.de

${ }^{\dagger}$ These authors contributed equally to this work.

Published by the American Physical Society under the terms of the Creative Commons Attribution 4.0 International license. Further distribution of this work must maintain attribution to the author(s) and the published article's title, journal citation, and DOI.
More generally, the concept of topology brought a unique understanding of the physics of materials, such as for topological insulators that are insulating in the bulk, yet have conducting surface and edge states [4]. Other examples are superconducting (SC) junctions made of nanowires that have opened the path to engineering topological superconductors [5]. Such SC systems can host exotic states, the so-called Majorana zero-energy modes, which have attracted broad interest fueled by the promise of topologically protected quantum computing [6-9]. More specifically, Majorana-based qubits illustrate the idea of holonomic quantum computation in a paradigmatic way that is based on the concept of a generalized non-Abelian Berry phase in a degenerate ground-state subspace [10]. In this scheme, after an adiabatic and cyclic change of the system's parameters, the initial state in the ground-state subspace can be transformed to a linear combination of the subspace basis that depends on the evolution of the parameters. Thus, adiabatic cyclic paths implement arbitrary unitary transformations - or quantum gates in the language of quantum information - in the degenerate subspace $[11,12]$, whose speed is limited by the inverse of the energy difference with the first excited state (or energy gap) [13].

The continuous search for new types of topological quantum matter has recently led to the discovery of topologically nontrivial quantum states in conventional multiterminal Josephson junctions [14-21]. Because of their 
scalability, SC Josephson circuits have already opened the path towards realistic implementations of quantum technologies [22,23]. These systems are based on the Josephson effect $[24,25]$ in which a supercurrent flows through a weak link that can be a tunnel junction, a molecule, or a quantum dot between two superconductors [26-29]. Microscopically, this supercurrent is carried by the so-called Andreev bound states that are localized at the weak link [30]. Because they have discrete energies inside the SC gap, Andreev bound states can be coherently manipulated and experimentally accessed by microwave spectroscopy [31-34] and supercurrent spectroscopy [35]. Andreev bound states can be exploited to encode information in novel types of SC qubits that are not based on collective electromagnetic degrees of freedom, such as charge, SC phase, or flux, but on microscopic quasiparticle states inherent to SC weak links [36,37]. At the same time, such systems, as for example multiterminal SC Josephson junctions, have turned out to be an ideal platform where synthetic topological materials can be engineered almost at will [14-21].

Interestingly, topology is not restricted to lowdimensional systems as the canonical example of the 2D quantum Hall effect, but it can also emerge in higherdimensional spaces in which controlling parameters play the role of extra synthetic dimensions. The intriguing case is if a system may be topologically trivial within a restricted (2D) subspace (namely it has a vanishing first Chern number) yet can show nontrivial topology in higher dimensions with nonzero higher-dimensional invariants (e.g., a nonzero second Chern number in 4D space). Indeed, the concept of the 2D quantum Hall effect has been extended to the 4D quantum Hall effect with the quantized nonlinear Hall response determined by the second Chern number [38]. Even though this situation does not naturally arise in solid-state systems due to limited dimensionality, there are several possibilities to create the 4D space artificially. It has been theoretically proposed to implement the 4D quantum Hall effect using the internal transitions of cold atoms in a 3D optical lattice [39] or in a 2D crystal with modulated on-site potentials [40]. The latter proposal has been experimentally realized in a system composed of an angled optical superlattice of ultracold bosonic atoms [41] and in tunable 2D arrays of photonic waveguides [42]. Other theoretical proposals to explore topological higher-dimensional systems are based on the simulation of a non-Abelian Yang monopole by cyclically coupling the hyperfine structure of rubidium [43] and on the implementation of one-way optical waveguides with designed spatial modulations [44]. Finally, even topologically protected Majorana modes can arise from a nontrivial second Chern number [45].

Overall, so far, a very limited number of implementations of higher-dimensional topological systems have been theoretically proposed and only a few of them have been experimentally realized. In this work, we show that nanoscale SC systems can implement higher-dimensional topology and represent a formidable platform to study a quantum system with a nontrivial second Chern number. In our proposal, the order is not limited by spatial dimensions since the additional dimensions can be easily implemented by increasing the number of independent SC phases applied at different SC contacts. We propose systems formed by multiterminal SC contacts embedding quantum dots and that are characterized by a nontrivial second Chern number. In the topological regime, for a large energy gap and perturbative tunneling coupling between the SC leads, the system possesses one pair of twofold degenerate states with a non-Abelian Berry phase. We show that the nontrivial 4D topology manifests itself in the integrated microwave response of the system by means of nonadiabatic effects [46]. We present a method to measure the non-Abelian Berry curvature in the degenerate subspace using a suitable measurement protocol. First, we discuss a deterministic scheme for the initial preparation of a target state in the ground-state subspace. The scheme is based on the adiabatic sweep from the nondegenerate case to the degenerate case followed by specific adiabatic cyclic paths (non-Abelian Berry rotations) of the SC phases. Since microwave spectroscopy on discrete Andreev bound states is a well-established technique by now [31-34], as a second step we apply designed polarized microwave spectroscopy [20] to access the diagonal and off-diagonal elements of the non-Abelian Berry curvature in the degenerate subspace. Finally, the difference in the oscillator strengths for different circular polarizations integrated over the 4D parameter space corresponds to the integration of the local Berry curvature and, therefore, the result will be directly related to the second Chern number.

\section{MODEL AND RESULTS}

\section{A. Model system consisting of two quantum dots}

Our starting point are two quantum dots (left $L$ and right $R$ ) with two spin levels whose average energies are $\epsilon_{L}$ and $\epsilon_{R}$, respectively. A local Zeeman field $B_{z}$ is externally applied with opposing direction on each dot. Experimentally, this opposing magnetic field could be realized with a multilayer magnetic texture in a similar scheme as discussed in Ref. [47]. Such a simple double-dot system is coupled to a network composed of different SC contacts that we call hereafter the environment. For the sake of simplicity, we assume that all the SC contacts are similar, namely with the same SC gap $\Delta$ and at the same chemical potential set to zero as a reference. The SC phases associated with the environment provide the synthetic gauge fields composed of four independent parameters $\lambda=\left(\lambda_{1}, \lambda_{2}, \lambda_{3}, \lambda_{4}\right)$ from which we define topological properties. The independent phases are used to implement a four-dimensional space providing nontrivial topology in 
terms of higher-dimensional invariants. Indeed, after integrating out the environment, the tunnel coupling of the dots with the SC environment results in an effective phasedependent Hamiltonian acting solely on the double-dot system that defines the energy spectrum of the lowest discrete states inside the SC gap. Naturally, the final result depends on the structure of the environment and what kind of interaction or structure we synthesize in the double-dot system. The advantage of this general method is the large freedom in constructing a specific effective Hamiltonian of the double-dot system providing all the necessary extra dimensions.

As depicted in Fig. 1(a), the minimal effective Hamiltonian of the double-dot system showing a nontrivial second Chern number has the form

$$
\begin{aligned}
H= & \sum_{\alpha=L, R} \sum_{\sigma=\uparrow, \downarrow} \epsilon_{\alpha} d_{\alpha \sigma}^{\dagger} d_{\alpha \sigma} \\
& +\left[B_{z}+Z(\lambda)\right] \sum_{\sigma=\uparrow, \downarrow} \sigma\left(d_{L \sigma}^{\dagger} d_{L \sigma}-d_{R \sigma}^{\dagger} d_{R \sigma}\right) \\
& +\sum_{\sigma=\uparrow, \downarrow}\left[K(\lambda) d_{L \sigma}^{\dagger} d_{R \sigma}+\text { H.c. }\right] \\
& +\left[F(\lambda)\left(d_{L \uparrow}^{\dagger} d_{L \downarrow}-d_{R \uparrow}^{\dagger} d_{R \downarrow}\right)+\text { H.c. }\right]
\end{aligned}
$$

where $d_{\alpha \sigma}^{\dagger}\left(d_{\alpha \sigma}\right)$ is the creation (annihilation) operator of an electron with spin $\sigma=\uparrow, \downarrow$ on the left $(\alpha=L)$ or the right $(\alpha=R)$ dot, respectively, and H.c. denotes the Hermitian conjugate. As anticipated, the effective Hamiltonian possesses an additional parameter-dependent Zeeman term $Z(\lambda)$ on both dots with reversed direction. As shown in Fig. 1(b), such a Hamiltonian provides a twofold degenerate ground state $\left|E_{-}^{(1 / 2)}\right\rangle$ and a twofold degenerate excited state $\left|E_{+}^{(1 / 2)}\right\rangle$ at the symmetric point $\epsilon_{L}=\epsilon_{R}=\epsilon_{0}$ with energies $\quad E_{ \pm}=\epsilon_{0} \pm \sqrt{\left|B_{z}+Z(\lambda)\right|^{2}+|K(\lambda)|^{2}+|F(\lambda)|^{2}}$. To engineer this Hamiltonian, we need a parameterdependent coupling between both dots via the complex hopping $K(\lambda)$. Finally, we also demand a complex parameter-dependent spin-flip term $F(\lambda)$ on each dot with reversed sign between the left and right dots. We show how to engineer these terms using the SC multiterminal environment in Fig. 2 by coupling the SC leads and the quantum dots via normal single-electron tunneling [29], and with normal or spin-orbit coupled SC leads. The latter coupling was recently revealed in Josephson matter devices $[33,34]$ and should be, in principle, experimentally feasible. For instance, as depicted in Figs. 2(a)-2(c), an effective phase-dependent hopping $K \propto e^{i\left(\phi_{2}-\phi_{1}\right)}$ between the two dots can be realized via two crossed Andreev reflection (CAR) processes in which Cooper pairs break in one lead and recombine in another lead, effectively transferring an electron from one to the other dot. Similarly, as shown in Figs. 2(d)-2(f), one can also engineer a phase-dependent effective local spin flip $F \propto \sin \left(\phi_{2}-\phi_{1}\right)$ with the help of two spin-orbit coupled leads where again two CAR processes are involved. Finally, an effective phase-dependent local Zeeman splitting $Z \propto \sin \left(\phi_{1}-\phi_{2}\right)$ on the dots is realized via a spin-dependent hopping between two SC leads that are coupled to the same dot; see Figs. 2(g)-2(i). For the sake of simplicity, $K, F, Z$ are evaluated here in the lowest order of the tunnel coupling between the SC leads. Taking higher orders will lead to small corrections from the sinusoidal dependence; however, this does not interfere with the validity of the statements in the following sections.

The Hamiltonian in Eq. (1) conserves the fermionic parity. Assuming strong Coulomb interactions on the dots, local Andreev reflection, in which a single Cooper pair is injected from an SC lead to a single dot, is forbidden. This (a)

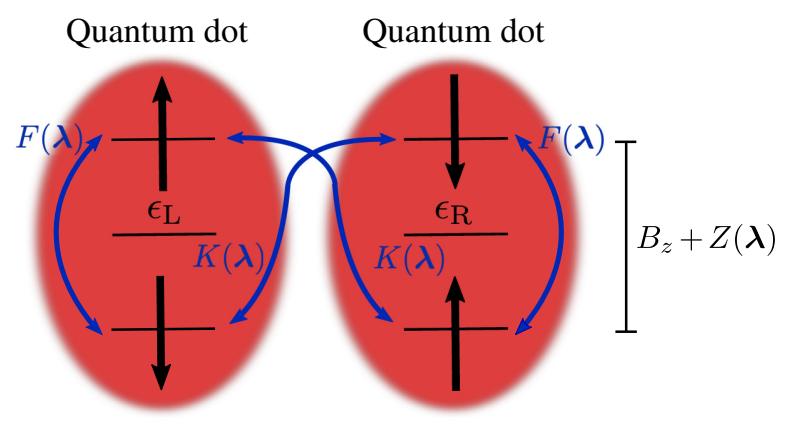

(b) $\epsilon_{\mathrm{L}} \neq \epsilon_{\mathrm{R}}$

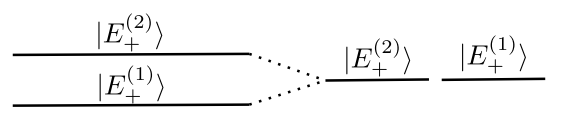

(c)

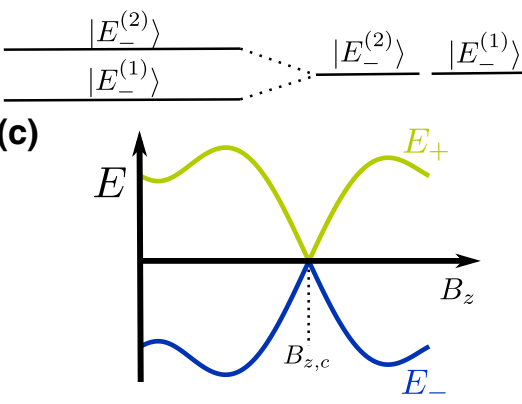

FIG. 1. (a) Double-quantum-dot system with mediated interactions $F(\lambda), K(\lambda)$, and $Z(\lambda)$ via four parameters $\lambda=\left(\lambda_{1}, \lambda_{2}, \lambda_{3}, \lambda_{4}\right)$. (b) For $\epsilon_{L} \neq \epsilon_{R}$, there are two pairs of states in the double-dot system. At the symmetric point for $\epsilon_{L}=\epsilon_{R}$, there is only one pair of twofold degenerate states. (c) Sketch of an energy crossing of the two twofold degenerate bands appearing at the crossing point $B_{z, c}$ for $\lambda=\lambda_{c}$, which leads to a change of the second Chern number. 
(a)

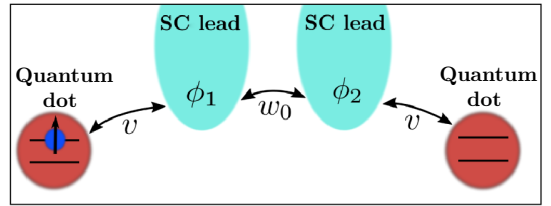

(d)

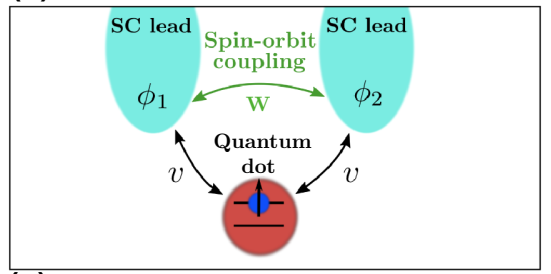

(g)

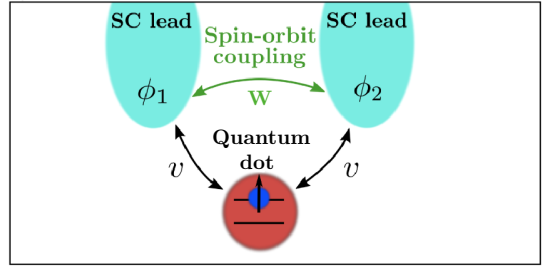

(b)

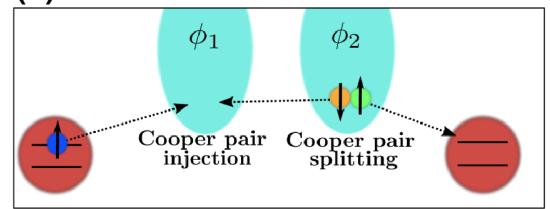

(e)

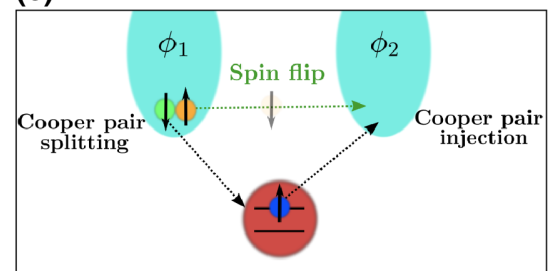

(h)

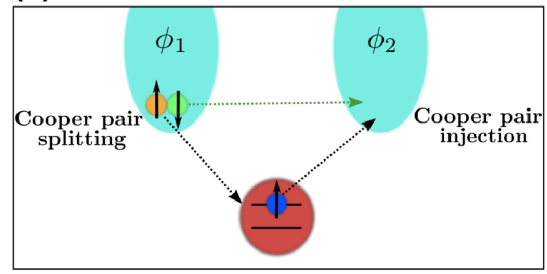

(c)

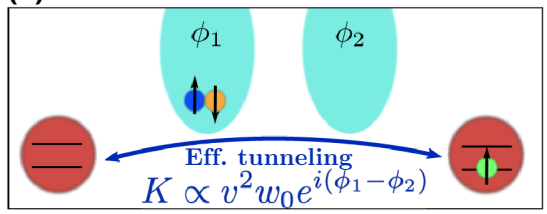

(f)

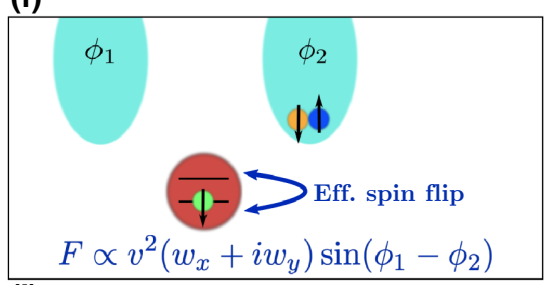

(i)

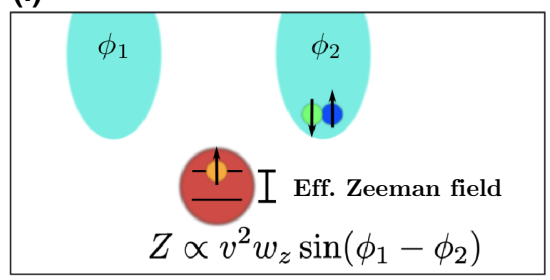

FIG. 2. (a) Two dots (red) coupled to two SC leads with $w_{0} \ll v$. (b) The effective hopping between the dots is mediated by two crossed Andreev reflection processes in which a Cooper pair is combined (left lead) and one Cooper pair is split (right lead). (c) This mechanism leads to an effective tunneling $K \propto v^{2} w_{0} e^{i\left(\phi_{1}-\phi_{2}\right)}$ depending on the two phases of the leads. (d) One dot (red) coupled to two SC leads that are themselves connected via spin-orbit coupling $\mathbf{w}$. (e) The spin-flip of an electron on the dot is mediated by two crossed Andreev reflection processes in which a Cooper pair is combined (right lead) and one Cooper pair is split (left lead). For this mechanism, a Rashba-like spin-orbit interaction between the superconductors is needed. (f) The process results in an effective phase-dependent spin-flip term $F \propto v^{2}\left(w_{x}+i w_{y}\right) \sin \left(\phi_{1}-\phi_{2}\right)$ on the dot. (g) One dot (red) coupled to two SC leads that are themselves coupled via spin-orbit interaction w. (h) The mechanism is mediated by two crossed Andreev reflection processes in which a Cooper pair is combined (right lead) and one Cooper pair is split (left lead). (i) The process results in an effective Zeeman term $Z \propto v^{2} w_{z} \sin \left(\phi_{1}-\phi_{2}\right)$ on the dot.

allows us to analyze the Hamiltonian in Eq. (1) by focusing only on the odd-parity regime in which the full double-dot system is occupied by only a single electron. In the following, as a proof of concept, we give two examples for the SC environments that allow for the presented structure and lead to a nontrivial second Chern number. Although this scheme is totally general, we present two specific examples to illustrate the underlying ideas.

\section{Example A}

A first example of a topological higher-dimensional system is formed by a multiterminal structure composed of nine standard BCS SC contacts, as shown in Fig. 3(a). In general, each $\mathrm{SC}$ contact provides an individual phase. However, by connecting neighboring SC terminals to form $\mathrm{SC}$ loops, the individual phase differences can be experimentally fixed and tuned by magnetic fluxes in the way we propose $[30-35,48,49]$. Whereas gauge invariance allows us to set one phase to zero $\left(\phi_{0}=0\right)$ as a reference, we set the other phases to values such that only four SC phases are independent; see Fig. 3(a). Hence, the parameter space is defined by these four phases, i.e., $\lambda_{A}:=\left(\phi_{1}, \phi_{2}, \phi_{3}, \phi_{4}\right)$.
As shown in Fig. 3(a), some contacts are tunnel coupled to the left dot or the right dot and some contacts are also tunnel coupled between each other. In particular, the SC leads are either coupled via normal tunnel hoppings, described by a scalar parameter $w_{0}$ taking the form $w_{0}\left(\sigma_{0} \otimes \tau_{3}\right)$ in spin (Pauli matrices $\left.\sigma_{0}, \ldots, \sigma_{3}\right)$ space and Nambu (Pauli matrices $\tau_{0}, \ldots, \tau_{3}$ ) space, respectively, or coupled via spin-orbit tunnel coupling, described by the vectors $\mathbf{w}_{j}=\left(w_{x, j}, w_{y, j}, w_{z, j}\right)^{\top}$, namely spin-flip processes are possible during the tunneling. Here, the tunneling can be expressed as $i \mathbf{w}_{j} \cdot \sigma \otimes \tau_{3}$ in spin-Nambu space (see Appendix A). Assuming large local Coulomb interactions on the dots, Cooper pair injections are excluded and, in the odd-parity subspace, the resulting low-energy Hamiltonian for the lowest pair of states takes the form of Eq. (1) to first order in the coupling between the leads $\left(w_{0},\left|\mathbf{w}_{j}\right| \ll v\right)$. Here, $v$ is the normal coupling of the dots to the environmental leads. The explicit form of the terms reads (derived in Appendix A)

$$
\begin{aligned}
& Z\left(\lambda_{A}\right)=-2 \epsilon_{T} R_{z}\left(\lambda_{A}\right), \\
& K\left(\lambda_{A}\right)=v_{0}-\epsilon_{T}\left(e^{i \phi_{1}}+e^{-i \phi_{2}}\right),
\end{aligned}
$$




$$
F\left(\lambda_{A}\right)=-2 \epsilon_{T}\left[R_{x}\left(\lambda_{A}\right)-i R_{y}\left(\lambda_{A}\right)\right]
$$

where $v_{0}$ is the direct coupling between the dots and $\epsilon_{T}=$ $\pi^{2} N_{0}^{2} v^{2} w_{0}$ is the characteristic energy of the mediated hopping by the superconductors. In addition, $N_{0}$ is the normal density of states at the Fermi energy of the superconductors. To give an estimation, we expect the characteristic energy $\epsilon_{T}$ of this process to be of the order of several tens of microelectronvolts when comparing to experimental coupling energies between quantum dots and superconducting leads (e.g., Refs. [50,51]). All following energy scales are reported in respect to this energy. The dimensionless vector $\mathbf{R}\left(\lambda_{A}\right)=\left[\mathbf{w}_{1} \sin \left(\phi_{3}\right)+\mathbf{w}_{2} \sin \left(\phi_{4}-\phi_{3}\right)+\right.$ $\left.\mathbf{w}_{3} \sin \left(\phi_{4}-\phi_{2}\right)\right] / w_{0}$ is associated with the spin-orbit tunnel hopping. A more detailed discussion on the range of parameters and the stability of the topological phase is presented in Appendix B.

\section{Example B}

As a second example, we consider an environment with eight leads, as depicted in Fig. 3(b). Four leads will be standard BCS superconductors described by the two SC phases $\phi_{1}$ and $\phi_{0}$ with $\phi_{0}=0$ as our choice of gauge. These leads are coupled either by a spin-dependent hopping $\left(\sigma w_{0} e^{i \pi / 2}\right)$ with a complex phase or by a simple tunnel coupling (w), as illustrated in Fig. 3(b). The other four leads are assumed to be superconductor-ferromagnet (SFM) hybrid bilayers [52]. The latter contacts are described by the two SC phases $\phi_{2}$ and $\phi_{0}=0$. In addition, the local magnetizations are assumed to have the same magnitude $h$, but are tilted by relative angles $\theta_{j}$ with respect to the quantization axis associated with the Zeeman field $B_{z}$ in the dots. Therefore, the parameter space for Example $\mathrm{B}$ is given by $\lambda_{B}:=\left(\phi_{1}, \phi_{2}, \theta_{1}, \theta_{2}\right)$. The superconductorferromagnet hybrid bilayer contacts are coupled via normal hoppings $\left(\tilde{w}_{0}\right)$. Again, in the first order of the coupling between the leads $\left(w_{0} \ll v, \tilde{w}_{0} \ll \tilde{v}\right)$ and in the odd-parity regime (large local Coulomb interactions), the structure of the double-dot system takes the form of Eq. (1) with the terms

$$
\begin{aligned}
Z\left(\lambda_{B}\right)= & \frac{h \epsilon_{h}}{\sqrt{\Delta^{2}-h^{2}}}\left[\cos \left(\theta_{1}\right)+\cos \left(\theta_{2}\right)\right]-2 \epsilon_{T} \sin \left(\phi_{1}\right) \\
K\left(\lambda_{B}\right)= & -\left(\epsilon_{T} e^{i \phi_{1}}+\frac{\tilde{\epsilon}_{T}\left(h^{2}+\Delta^{2} e^{-i \phi_{2}}\right)}{\Delta^{2}-h^{2}}\right) \\
F\left(\lambda_{B}\right)= & \frac{h \epsilon_{h}}{\sqrt{\Delta^{2}-h^{2}}}\left[\sin \left(\theta_{1}\right)+\sin \left(\theta_{2}\right)\right] \\
& +i \frac{2 h^{2}}{\Delta^{2}-h^{2}} \tilde{\epsilon}_{T} \sin \left(\theta_{2}-\theta_{1}\right)
\end{aligned}
$$

as derived in Appendix $\mathrm{C}$ with $\tilde{\epsilon}_{T}=\pi^{2} N_{0}^{2} \tilde{v}^{2} \tilde{w}_{0}$ and $\epsilon_{h}=\pi N_{0} \tilde{v}^{2}$, whereas $\Delta$ is the gap of the superconductors. The energy of both dots are renormalized by $\tilde{\epsilon}_{0}=\epsilon_{0}-\Delta^{2} \tilde{\epsilon}_{T} \sin \left(\phi_{2}\right) /\left(\Delta^{2}-h^{2}\right)$. However, this renormalization does not influence our topological findings since the discrete low-energy spectrum of the system is still given by the eigenvalues $E_{ \pm}=\tilde{\epsilon}_{0} \pm$ $\sqrt{\left|B_{z}+Z\left(\lambda_{B}\right)\right|^{2}+\left|K\left(\lambda_{B}\right)\right|^{2}+\left|F\left(\lambda_{B}\right)\right|^{2}}$. A more detailed discussion on the range of parameters and the stability of the topological phase is presented in Appendix D.

\section{B. Second Chern number}

The topological information of the system is encoded in the non-Abelian Berry curvature $[53,54]$ defined as $F_{j k}=\partial_{j} A_{k}-\partial_{k} A_{j}-i\left[A_{j}, A_{k}\right]$, where $A_{j}$ is the matrixvalued non-Abelian Berry connection that has the elements $\left[A_{j}\right]_{\alpha \beta}=i\left\langle\psi_{\alpha} \mid \partial_{j} \psi_{\beta}\right\rangle$ calculated from the eigenstates $\left|\psi_{\alpha}\right\rangle$ of the degenerate energy levels and $\partial_{j}=\partial / \partial \lambda_{j}$. The first Chern number takes the usual form $C^{\left(1_{j k}\right)}=$ $(1 / 2 \pi) \int_{\mathbb{T}^{2}} d^{2} \lambda \operatorname{Tr}\left(F_{j k}\right)$ when integrating the Berry curvature over the 2-torus $\mathbb{T}^{2}$ spanned by the $2 \mathrm{D}$ phase space $\left(\lambda_{j}, \lambda_{k}\right)$, and the trace is taken with respect to the degenerate basis states. However, it turns out that in our model with a pair of two degenerate levels, the first Chern number always vanishes [43,55], $C^{\left(1_{j k}\right)}=0$.

Remarkably, the system is still topological in the sense of a higher-dimensional invariant. In the four-dimensional space, we consider the second Chern number $[56,57]$

$$
C^{(2)}=\frac{1}{32 \pi^{2}} \int_{\mathbb{T}^{4}} d^{4} \lambda \varepsilon^{j k l m} \operatorname{Tr}\left(F_{j k} F_{l m}\right),
$$

with the integration over the 4-torus $\mathbb{T}^{4}$ defined by the compact parameters $\left(\lambda_{1}, \lambda_{2} . \lambda_{3}, \lambda_{4}\right)$. Here, $\varepsilon^{j k l m}$ is the LeviCivita symbol in four dimensions and the sum runs over repeated indices. It is worth noting that, in contrast to the first Chern number that is solely determined by the diagonal components of the Berry curvature, we need additional information on the off-diagonal elements for the second Chern number.

In the following, we show that the second Chern number does not vanish, $C^{(2)} \neq 0$, for some range of parameters of the system. An advantageous property exists for the symmetric case $\epsilon_{L}=\epsilon_{R}=\epsilon_{0}$. Under this condition, the effective Hamiltonian in Eq. (1) can also be expressed by means of a set of five anticommuting Dirac matrices $\boldsymbol{\Gamma}=$ $\left(\Gamma_{1}, \ldots, \Gamma_{5}\right)$ with which we can write $H=\mathbf{d}^{\dagger}\left[\epsilon_{0} \mathbb{1}+\boldsymbol{\Gamma}\right.$. $\mathbf{H}(\lambda)] \mathbf{d}$, where the spinor of the dot operators reads $\mathbf{d}^{\dagger}=$ $\left(d_{L \uparrow}^{\dagger}, d_{L \downarrow}^{\dagger}, d_{R \uparrow}^{\dagger}, d_{R \downarrow}^{\dagger}\right)$. Here, $\mathbb{1}$ is a $4 \times 4$ unit matrix and the Hamiltonian describes a pseudospin $\Gamma$ in an effective magnetic field $\mathbf{H}(\boldsymbol{\lambda})=\left(H_{1}, H_{2}, H_{3}, H_{4}, H_{5}\right)$, where $H_{1}=$ $B_{z}+Z, H_{2}=\operatorname{Re}[K], H_{3}=\operatorname{Im}[K], H_{4}=\operatorname{Re}[F]$, and $H_{5}=$ $\operatorname{Im}[F]$. Using this representation, the second Chern number can be written as a winding number of the $4 \mathrm{D}$ hypersurface 
(a)

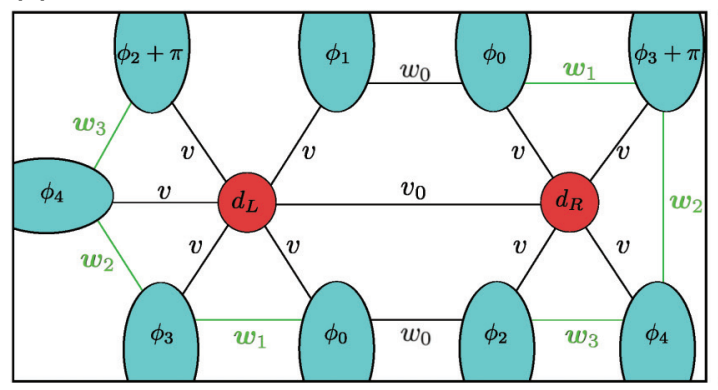

(b)

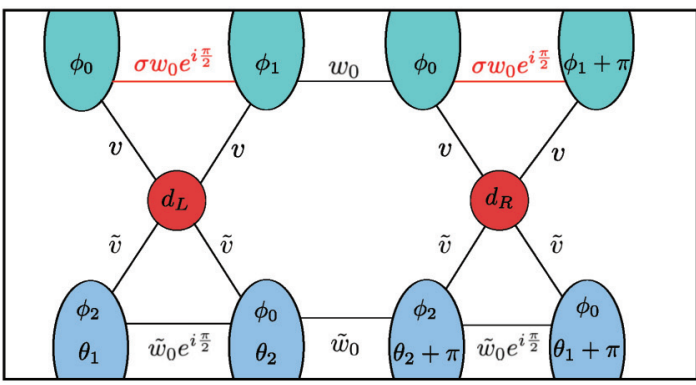

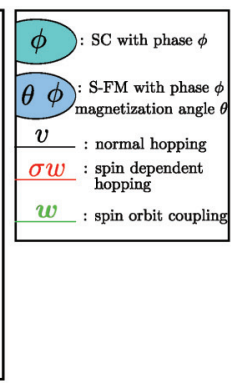

FIG. 3. (a) Full system of Example A with an environmental network of nine SC leads coupled via normal hoppings $\left(w_{0}, v_{0}, v\right)$ or via spin-orbit couplings $\left(\mathbf{w}_{j}\right)$. Each lead has a SC phase $\phi_{j}$ and the dots have a magnetic field $B_{z}$ in opposing directions. Note that the phases of each lead can be controlled by connecting neighboring leads via SC loops. Since the fluxes through these loops can be independently controlled, the phases are effectively independent of its neighbors and can be tuned to the proposed setup [30-35,48,49]. (b) Full system of Example B with an environmental network of four SC leads with normal hopping $\left(v, w_{0}\right)$ and spin-dependent hopping $(\sigma w)$, as well as four SC leads with a local exchange field $h$ with magnetization direction $\theta_{j}$ (with respect to the direction of a magnetic field $B_{z}$ on the dots) coupled via normal hopping $\left(\tilde{v}, \tilde{w}_{0}\right)$. Each lead has a SC phase $\phi_{j}$ and the dots have a magnetic field $B_{z}$ in opposing directions.

traced out by $\mathbf{H}(\lambda)$ about the origin, i.e.,

$$
C^{(2)}=\frac{3}{8 \pi^{2}} \int_{\mathbb{T}^{4}} d^{4} \lambda \varepsilon^{j k l m n} \frac{H_{j}\left(\partial_{1} H_{k}\right)\left(\partial_{2} H_{l}\right)\left(\partial_{3} H_{m}\right)\left(\partial_{4} H_{n}\right)}{|\mathbf{H}|^{5}},
$$

counting how often the map $\mathbf{n}=\mathbf{H} /|\mathbf{H}|$ wraps around the unit sphere [56]. Here, $\varepsilon^{j k l m n}$ is the Levi-Civita symbol in five dimensions and the sum runs over repeated indices. Using the expression in Eq. (5), we numerically calculate the second Chern number using the Monte Carlo method [58] and report the results in Fig. 4(a) for Examples A and $\mathrm{B}$ as a function of the externally applied magnetic field $B_{z}$. We see that there are different topological phases with nonzero values of the second Chern number for both systems. Interestingly, Example A admits a nontrivial second Chern number even in the absence of the external magnetic field $B_{z}=0$. It is worth analyzing the behavior of the two energies associated with the twofold degenerate states, namely $E_{-}$for the ground states and $E_{+}$for the excited states. These two energies are two effective energy bands in a 4D space with the compact parameters $\lambda_{1}, \lambda_{2}, \lambda_{3}, \lambda_{4}$ playing the role of generalized quasimomenta in a first Brillouin zone. Similarly to the behavior occurring in 2D topological systems characterized by the first Chern number, the topological transition with the concomitant discontinuous change of the second Chern number is signaled by the occurrence of crossing points between the two isolated bands $E_{-}$and $E_{+}$in the 4 D space. In our effective model Hamiltonian describing the double-dot system, the upper band $E_{+}$and the lower band $E_{-}$have crossing points only if $|\mathbf{H}|=0$. This equation defines the critical magnetic field $B_{z, c}$ as well as the critical values for the parameters $\lambda_{c}=\left(\lambda_{1, c}, \lambda_{2, c}, \lambda_{3, c}, \lambda_{4, c}\right)$. In order to illustrate this issue, we show the energies $E_{+}$and $E_{-}$in Fig. 4(b) for Example B as a function of the two parameters $\lambda_{3}, \lambda_{4}$ at a fixed value of the other two parameters $\lambda_{1, c}=\lambda_{2, c}=$ $\arccos (-1 / 8)$. Note that the two bands cross at the point $\lambda_{3, c}=\lambda_{4, c}=\pi$ with nonvanishing first derivatives. Furthermore, the crossing does not happen at zero energy since the energy levels are renormalized in Example B.

\section{Non-Abelian Berry rotations in a degenerate subspace}

A cyclic change of the parameters $\lambda(t)$ over time $t$ defining a periodic path in parameter space is adiabatically slow if the characteristic scale variations of the parameters $\partial \lambda_{j} / \partial t(j=1, \ldots, 4)$ remain much smaller than the energy gap $\left(E_{+}-E_{-}\right) \gg \hbar\left|\partial \lambda_{j} / \partial t\right|$ between the first excited state with energy $E_{+}$and the ground state with energy $E_{-}$(with $\hbar$ being Planck's constant). If the ground state is nondegenerate, the final state acquires a complex geometric phase, known as the Berry phase [53], at the end of the cyclic path. For a degenerate ground state, the non-Abelian Berry phase due to a cyclic path represents a unitary transformation of the initial state in the degenerate subspace $[10,11]$. In our system, the ground state is spanned by two eigenstates and the cyclic path results in a rotation in this two-dimensional degenerate subspace. The adiabatic condition guarantees that the final state has vanishing components in the twofold degenerate excited state after the cyclic path, namely Landau-Zener transitions are negligible. Consider the closed path $C_{p}: t \mapsto$ $\lambda^{(p)}(t)$ for the time range $t \in[0, T]$ with the periodic condition $\lambda^{(p)}(0)=\lambda^{(p)}(T)$. Defining a specific basis in the ground-state subspace $\left|E_{-}^{(\alpha)}\right\rangle(\alpha=1,2)$ and assuming that the initial ground state is $\left|\Psi_{i}\right\rangle=\left|E_{-}^{(1)}\right\rangle$, the final ground 
(a)
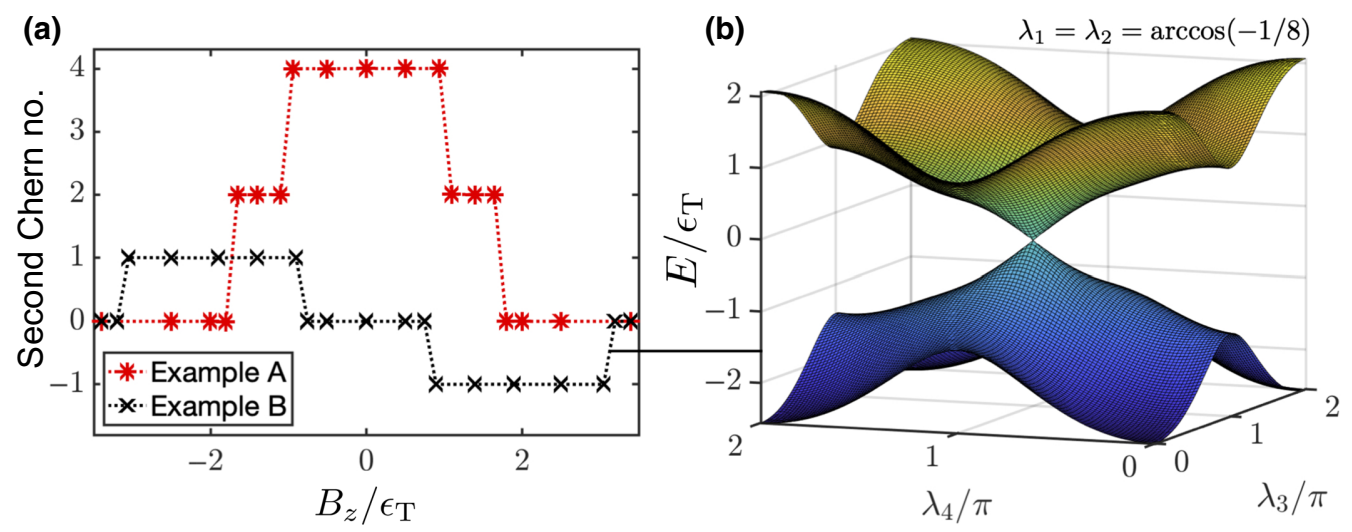

FIG. 4. (a) Second Chern number numerically evaluated with Monte Carlo integration for $N=10^{9}$ points in $\lambda$ space. In Example A, we set $v_{0}=\epsilon_{T}, \mathbf{w}_{1} / w_{0}=(-0.5,0,0.5), \mathbf{w}_{2} / w_{0}=(0,0.5,1)$, and $\mathbf{w}_{3} / w_{0}=(0.5,0,0.5)$. In Example $\mathrm{B}$, we set $\epsilon_{T}=4 \tilde{\epsilon}_{T} / 3=\epsilon_{h}$ and $h=\Delta / 2$. (b) Energy bands with a crossing point for Example B at $B_{z} \approx 3.14 \epsilon_{T}$, which changes the second Chern number from -1 to 0 . In both cases $\epsilon_{L}=\epsilon_{R}=0$.

state $\left|\Psi_{f}\right\rangle$ after the evolution becomes the superposition

$$
\begin{aligned}
\left|\Psi_{f}\right\rangle= & \exp \left\{-\frac{i}{\hbar} \int_{0}^{T} d t^{\prime} E_{-}\left[\lambda^{(p)}\left(t^{\prime}\right)\right]\right\} \\
& \times\left(\gamma_{11}\left|E_{-}^{(1)}\right\rangle+\gamma_{21}\left|E_{-}^{(2)}\right\rangle\right),
\end{aligned}
$$

in which the first term is the (scalar) dynamical phase and the coefficients of the linear combination are given by the matrix elements of the unitary rotation as

$$
\gamma_{\alpha \beta}=\left[\mathcal{P} \exp \left(i \oint_{C_{p}} d \lambda \cdot \mathbf{A}_{-}(\boldsymbol{\lambda})\right)\right]_{\alpha \beta},
$$

namely the non-Abelian Berry phase describing the rotation. Here, $\mathbf{A}_{-}$is the non-Abelian Berry connection in the degenerate ground-state subspace and $\mathcal{P}$ is the pathordering operator. In general, due to the rotational character, the local and infinitesimal non-Abelian Berry rotations do not commute along the path $C_{p}$, which makes it necessary to use the path-ordering operator $\mathcal{P}$.

The preparation of the initial state can be simply achieved in the following way. Initially, one can introduce a small asymmetry $\epsilon_{L} \neq \epsilon_{R}$ in the two dots such that it lifts the degeneracy of the energy levels of the ground state in the symmetric case. This process unequivocally identifies the nondegenerate states $\left|E_{-}^{(1)}\right\rangle$ and $\left|E_{-}^{(2)}\right\rangle$ for $\epsilon_{L} \neq \epsilon_{R}$. After waiting a sufficiently long time, much larger than the time scale set by relaxation processes, one can ensure that the state is in the lowest state, which we label $\left|E_{-}^{(1)}\right\rangle$. Subsequently, the degeneracy is adiabatically restored by tuning $\epsilon_{L}=\epsilon_{R}$, such that the system remains in the $\left|E_{-}^{(1)}\right\rangle$ state. Then, one can make use of the non-Abelian Berry phase to create arbitrary rotations in the degenerate subspace by simply adiabatically changing the parameters along a closed loop. To give an idea of such implementations, we discuss in detail a state-preparation protocol example for the specific case corresponding to the system of Example A; see Fig. 3(a). In Fig. 5(a), we show two adiabatic cyclic paths in which two SC phases are always fixed, $\phi_{3}=\phi_{4}=0$, and we adiabatically change the other two phases $\phi_{1}$ and $\phi_{2}$. The first path is defined by the simultaneous change of the two phases with $\phi_{1}=\phi_{2}=\phi$ along the path $\phi=0 \rightarrow 2 \pi$, depicted in Fig. 5(a) as a black line. For the second path, depicted in Fig. 5(a) as a gray line, we only vary the phase $\phi_{1}$ and fix the other phase $\phi_{2}=0$. Representing the degenerate ground state on a Bloch sphere with the basis states $\left\{\left|E_{-}^{(1)}\right\rangle,\left|E_{-}^{(2)}\right\rangle\right\}$, these two adiabatic cyclic paths implement qubit rotations, as presented in Fig. 5(b). While the first black path results in a rotation described by the polar angle $\theta$, the second gray path yields a rotation in the azimuthal direction described by the angle $\varphi$, where both values of the angles $\theta$ and $\varphi$ depend on the value of the externally applied magnetic field $B_{z}$ in the two dots, as shown in Fig. 5(b). With a desired combination of the two paths, achieved by previously fixing the externally applied magnetic field to the desired value, one can create every possible rotation of the $\mathrm{SU}(2)$ group in the degenerate subspace. It is also worth mentioning that it is possible to define other rotation axes on the Bloch sphere by choosing different adiabatic cyclic paths $C_{p}$ in parameter space along which Eq. (7) is evaluated. Similar results hold for Example B; see Fig. 3(b). In this case one can also vary the angles of the local magnetization in the hybrid superconductorferromagnet contacts, $\theta_{1}$ and $\theta_{2}$. For instance, the polar rotation is set by adiabatically varying $\theta_{1}$ and $\theta_{2}$ along the cyclic path defined by $\theta_{1}=\theta_{2}=0 \rightarrow 2 \pi$ and by setting the SC phases to $\phi_{1}=\phi_{2}=0$, whereas the azimuthal rotation is implemented by varying only $\phi_{2}=0 \rightarrow 2 \pi$ and by simply setting $\phi_{1}=\theta_{1}=\theta_{2}=0$. Clearly, the angle dependence on the local magnetic field $B_{z}$ is different in the case of Example B in comparison to Example A and depends on 
(a)

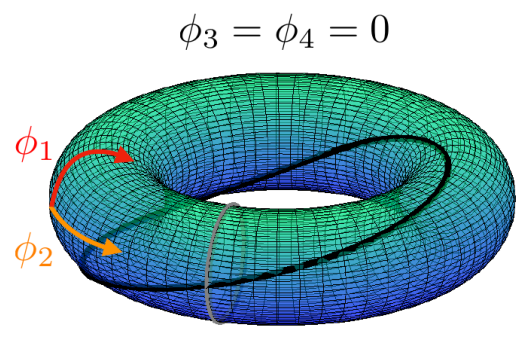

(b)

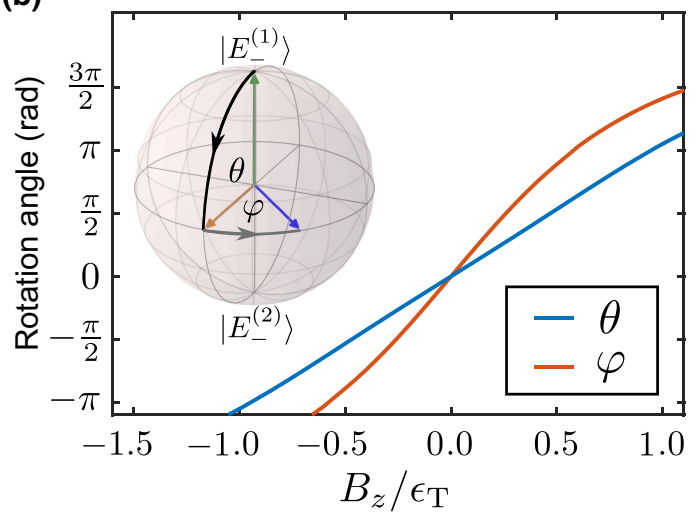

FIG. 5. (a) Protocol for qubit manipulations for any initial state [here $\left.\left|E_{-}^{(1)}\right\rangle\right]$ via the non-Abelian Berry phase in the case of Example A. The rotation from $\left|E_{-}^{(1)}\right\rangle$ to $\left|E_{-}^{(2)}\right\rangle$ (black) for the adiabatic change $\phi_{1}, \phi_{2}: 0 \rightarrow 2 \pi$ is depicted on a torus. A second rotation around the $z$ axis of the Bloch sphere (gray) can be achieved via the adiabatic change $\phi_{1}: 0 \rightarrow 2 \pi$ and $\phi_{2}=0 . \phi_{3}=\phi_{4}=0$ during the whole process. (b) Bloch sphere of the degenerate subspace $E_{-}$with the possible rotations in (a) (top-left corner). Numerically determined angles of the Berry rotations $\theta$ (by changing adiabatically $\phi_{1}, \phi_{2}: 0 \rightarrow 2 \pi$ ) and $\varphi$ (by changing adiabatically $\phi_{1}: 0 \rightarrow 2 \pi$ ) as a function of the magnetic field $B_{z}$ in the case of Example A for $v_{0}=\epsilon_{T}, \mathbf{w}_{1} / w_{0}=(-0.5,0,0.5), \mathbf{w}_{2} / w_{0}=(0,0.5,1), \mathbf{w}_{3} / w_{0}=(0.5,0,0.5)$, and $\epsilon_{R}=\epsilon_{L}=0$.

the concrete parameters of the setup. The rotation angles of Example B are further discussed in Appendix E.

\section{Measuring the second Chern number using polarized microwave spectroscopy}

We now discuss how to experimentally measure the second Chern number in higher-dimensional topological SC systems. The measurement scheme is based on microwave spectroscopy in which designed microwave excitations are applied to the system. In particular, we show how to get access to the local elements of the non-Abelian Berry curvature by means of polarized microwave spectroscopy from which the second Chern number automatically follows. This task corresponds to a nontrivial extension of the method proposed in SC multiterminal Josephson junctions with lower-dimensional topology, i.e., nonvanishing first Chern number, with the ground and excited states being nondegenerate [20].

Applying a small time-dependent periodic modulation of driving frequency $\omega$ to the two parameters $\lambda_{j}$ and $\lambda_{k}$ of the Hamiltonian in Eq. (1) and with a fixed phase difference $\delta$, we have

$$
H \rightarrow H+\frac{2 A}{\hbar \omega} \frac{\partial H}{\partial \lambda_{j}} \cos (\omega t)+\frac{2 A}{\hbar \omega} \frac{\partial H}{\partial \lambda_{k}} \cos (\omega t-\delta),
$$

with the condition of low microwave power $A \ll \hbar \omega$. To measure the non-Abelian Berry curvature, the transition rates for four initial states have to be extracted by analyzing the switching statistics of the single-photon absorption event [59]. In general, discrete lines appear in the microwave absorption spectrum originating from transitions from the ground-state subspace to the subspace of excited states under continuously applied radiation. For a weak perturbation, the intensity of these lines is quantified by the average transition rates, which can be computed using Fermi's golden rule following standard linear response theory, and by averaging over the initial and final states. However, by means of our preparation protocol discussed in Sec. II C, one can focus on a specific initial state before applying the microwave radiation, as illustrated in Fig. 6. As we show below, the non-Abelian Berry curvature will be related to this kind of transition. Therefore, we have to deal with different transition rates depending on the specific initial state and we have to average only over the final state. This information cannot simply be extracted from the microwave absorption spectrum and it is required to determine the average switching rate by preparing the same initial state many times.

Using the state preparation protocol defined in Sec. II C, one can univocally define the basis state in the ground and excited subspaces, which we denote by $\left|E_{ \pm}^{(\alpha)}\right\rangle(\alpha=1,2)$; see Fig. 6. Then, in order to measure the off-diagonal elements of the non-Abelian Berry curvature, we need to perform four different absorption measurements in which, for each of them, we have to initialize the system in the following four ground states: $\left|I_{1}\right\rangle=\left|E_{-}^{(1)}\right\rangle$ in Fig. 6(a), $\left|I_{2}\right\rangle=\left|E_{-}^{(2)}\right\rangle$ in Fig. 6(b), $\left|I_{3}\right\rangle=\left(\left|E_{-}^{(1)}\right\rangle+\left|E_{-}^{(2)}\right\rangle\right) / \sqrt{2}$ in Fig. 6(c), and $\left|I_{4}\right\rangle=\left(\left|E_{-}^{(1)}\right\rangle+i\left|E_{-}^{(2)}\right\rangle\right) / \sqrt{2}$ in Fig. 6(d). This is the minimal amount of measurements required to extract the non-Abelian Berry curvature [46]. Each state $\left|I_{\mu}\right\rangle(\mu=1,2,3,4)$ can be prepared using the previously discussed rotation protocols that are achieved 


\section{(a)

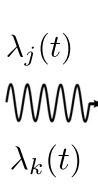

$\left|F_{1}\right\rangle$

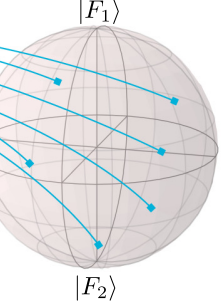

$\left|F_{1}\right\rangle$

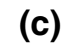

(c)

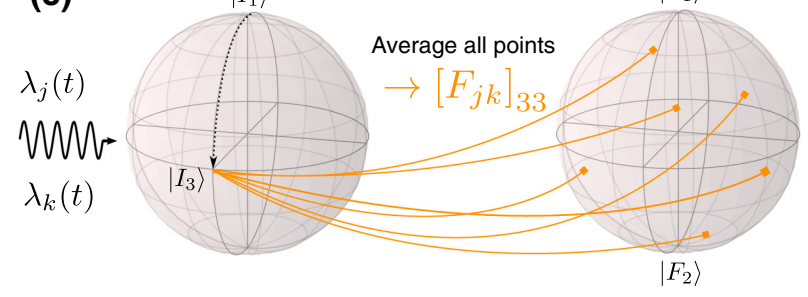

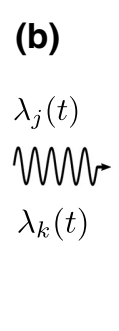

(d)

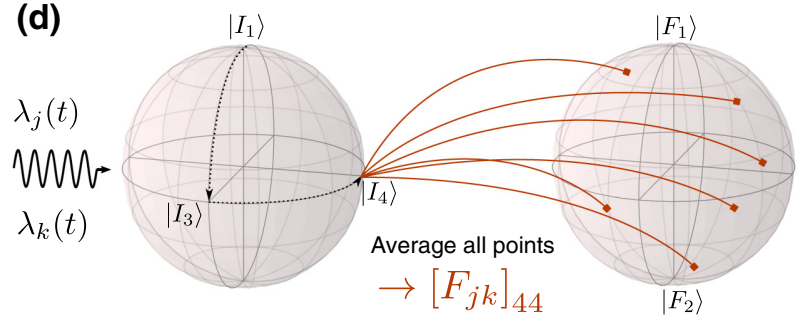

FIG. 6. (a),(b) Microwave spectroscopy protocol for the diagonal elements of the non-Abelian Berry curvature in the degenerate subspace. A small time-dependent modulation is applied to two parameters $\lambda_{j}(t)=2 A \cos (\omega t) / \hbar \omega \operatorname{and} \lambda_{k}(t)=2 A \cos (\omega t \pm \pi / 2) / \hbar \omega$, $j \neq k$, with $A / \hbar \omega \ll 1$, leading to a transition from the initial state $\left|I_{j}\right\rangle$ to an unknown final state $|F\rangle$. From the averaged response over the excited states, the diagonal part of the Berry curvature can be measured. Similarly, the diagonal Berry curvature with respect to the rotated states $\left|I_{3}\right\rangle$ (c) and $\left|I_{4}\right\rangle$ (d) can be measured. From these, the off-diagonal part of the non-Abelian Berry curvature with respect to the initial basis can be constructed by means of Eqs. (11).

by tailored adiabatic cyclic paths of the controlling parameters.

In general, applying the microwave signal at two parameters $\lambda_{j}$ and $\lambda_{k}$, as described in Eq. (8), results in a transition from the prepared initial state $\left|I_{\mu}\right\rangle$ to an arbitrary combination of the excited states, namely $|F\rangle=$ $\cos \left(\theta_{F} / 2\right)\left|E_{+}^{(1)}\right\rangle+\sin \left(\theta_{F} / 2\right) e^{i \varphi_{F}}\left|E_{+}^{(2)}\right\rangle$, as shown in Fig. 6, with the angles $\theta_{F}$ and $\varphi_{F}$ on the Bloch sphere of the excited subspace. The transition rates associated with the switching of the states are proportional to $[20,60]$

$$
\begin{aligned}
M_{j k, \mu}^{(\delta)}\left(\theta_{F}, \varphi_{F}\right)= & \left\langle I_{\mu}\left|\partial_{j} H\right| F\right\rangle\left\langle F\left|\partial_{j} H\right| I_{\mu}\right\rangle \\
& +\left\langle I_{\mu}\left|\partial_{k} H\right| F\right\rangle\left\langle F\left|\partial_{k} H\right| I_{\mu}\right\rangle \\
& +e^{i \delta}\left\langle I_{\mu}\left|\partial_{j} H\right| F\right\rangle\left\langle F\left|\partial_{k} H\right| I_{\mu}\right\rangle \\
& +e^{-i \delta}\left\langle I_{\mu}\left|\partial_{k} H\right| F\right\rangle\left\langle F\left|\partial_{j} H\right| I_{\mu}\right\rangle .
\end{aligned}
$$

One can measure the delay times from an application of the microwave drives until the system gets excited. By repeating this protocol several times and by analyzing the statistical distribution of these switching events, one can extract the average transition rates that are proportional to the matrix elements of Eq. (9). Finally, since we do not exactly know the final state, we consider the average of such matrix elements over all possible final states, namely over $\theta_{F}$ and $\varphi_{F}$. A priori, this should correspond to a real implementation of the measurement in which one repeats the single-photon absorption event several times.

As the matrix elements depend on the relative phase lag $\delta$ between the two modulations, one can choose $\delta=$ $+\pi / 2$ and $\delta=-\pi / 2$ to implement circularly polarized microwaves. The difference between these averaged matrix elements is related to the diagonal component of the local non-Abelian Berry curvature for the particular initial state $\left|I_{\mu}\right\rangle$, i.e.,

$$
\begin{aligned}
\frac{1}{4 \pi} \int_{0}^{\pi} d \theta_{F} \int_{0}^{2 \pi} d \varphi_{F} \sin \theta_{F}\left[M_{j k, \mu}^{(\pi / 2)}\left(\theta_{F}, \varphi_{F}\right)\right. \\
\left.-M_{j k, \mu}^{(-\pi / 2)}\left(\theta_{F}, \varphi_{F}\right)\right]=4 E_{-}^{2}\left[F_{j k}\right]_{\mu \mu}
\end{aligned}
$$

In this way, one has access to the diagonal components of the non-Abelian Berry curvature in the ground-state subspace with a basis of initial states with $\mu=1$ or $\mu=2$. The off-diagonal elements of the non-Abelian Berry curvature $\left[F_{j k}\right]_{12}$ and $\left[F_{j k}\right]_{21}$ in this basis can be obtained using the properties [46]

$$
\begin{aligned}
& {\left[F_{j k}\right]_{12}=\left[F_{j k}\right]_{33}-i\left[F_{j k}\right]_{44}-\frac{1-i}{2}\left(\left[F_{j k}\right]_{11}+\left[F_{j k}\right]_{22}\right),} \\
& {\left[F_{j k}\right]_{21}=\left[F_{j k}\right]_{33}+i\left[F_{j k}\right]_{44}-\frac{1+i}{2}\left(\left[F_{j k}\right]_{11}+\left[F_{j k}\right]_{22}\right),}
\end{aligned}
$$

in which $\left[F_{j k}\right]_{33}$ and $\left[F_{j k}\right]_{44}$ are given by Eq. (10) with the initial states $\mu=3$ and $\mu=4$. In simple words, one has to repeat the same measurement for all four different states in order to reconstruct all the elements of the non-Abelian Berry curvature matrix for a given modulation of $\lambda_{j}$ and $\lambda_{k}$.

In summary, one has to prepare the system in a state $\left|I_{\mu}\right\rangle$ with the help of the adiabatic cyclic path protocol presented in the previous section and apply circular 
microwave drives to $\lambda_{j}$ and $\lambda_{k}$ with $\delta= \pm \pi / 2$. The transition rates for the single absorption event are extracted by studying the switching statistics. By taking the difference between transition rates of left $(+\pi / 2)$ and right $(-\pi / 2)$ circularly polarized microwaves on the initial states for $\mu=1,2$, we obtain the diagonal elements of the Berry curvature $\left[F_{j k}\right]_{11}$ and $\left[F_{j k}\right]_{22}$, whereas the rate associated with the initial states $\mu=3,4$ allows us to reconstruct the off-diagonal elements $\left[F_{j k}\right]_{12}$ and $\left[F_{j k}\right]_{21}$ according to Eqs. (11). This has to be repeated for all $j, k=1,2,3,4$ with $j<k$ to find all Berry curvatures of the 4D parameter space $\lambda$, from which the second Chern number follows by integration according to Eq. (4).

As a final remark, note that if just a single parameter $\lambda_{j}$ is modulated or $\delta=0$ and $\delta=\pi$ are chosen, the groundstate metric tensor $g_{j k}$ can be measured as well; see Refs. $[20,60]$.

\section{DISCUSSION}

In this work, we have presented a first theoretical proposal for a nontrivial second Chern number in SC quantum systems, realizable via a double-dot system and an environmental network structure formed by SC contacts. We emphasize that the second Chern number can only be defined and studied in systems of dimension four or higher, which is realizable with the synthetic dimensions of the SC phases. Remarkably, the proposed system yields zero first Chern numbers, revealing the pure 4D nature of the topology.

The distinct topological phases with a nontrivial second Chern number, as shown in Fig. 4(a), should be robust against small fluctuations of the dot energies, possible random potentials, or thermal fluctuations, as long as the energy difference between the ground and excited states is large with respect to the energy scale of the fluctuations. However, closely around a topological phase transition point, which is marked by singular points in parameter space for which the energy gap is closed [cf. Fig. 4(b)], the second Chern number becomes particularly sensitive to fluctuations that could lead to small local deviations of the overall perfectly quantized behavior. Quasiparticle poisoning however could interfere with the proposed state preparation and measurement scheme, as it would introduce jumps in the parity destroying the coherence in the system. However, assuming an inter- and intradot Coulomb interaction, the system should be more robust to quasiparticle poisoning after being prepared in the oddparity regime. In similar schemes long parity lifetimes of up to a minute were observed [61].

We have furthermore shown that the parameter space is not necessarily restricted to SC phases only, as we have shown in Example B (cf. Fig. 3) in which a part of the space is analogously created by the magnetization angles of the leads. This yields a great freedom for eventually finding much simpler environmental networks with the presented structure in Eq. (1), where similar protocols for the non-Abelian Berry phase and the measurement of the second Chern number should hold as discussed in Secs. II C and II D, as they can be generally applied on such parameters. Furthermore, the non-Abelian Berry phase is potentially useful to create arbitrary rotations in a given subspace of degenerate quantum states, as shown in Fig. 5. This is a promising tool in this platform to manipulate the states for more detailed studies of higher-dimensional topological phases and is also needed for the proposed measurement scheme. Hence, a further direction of interest could be the stability of the non-Abelian Berry phase under local fluctuations of the system, which would define how stable and topologically protected these protocols for state manipulations would be in nonideal systems.

To conclude, we emphasize that this could stimulate further research activities for the investigation of higherdimensional topology and new types of topological phases in Josephson matter. This seems easily accessible in such systems due to the, in general, unlimited dimensionality, providing the potential to study even higher-dimensional problems [62].

\section{ACKNOWLEDGMENTS}

The authors are grateful for funding provided by the DFG through SFB 767 and Grant No. RA 2810/1.

Note added.-The authors became aware of several experimental implementations of non-Abelian Berry rotations [63-66].

\section{APPENDIX A. DERIVATION OF EXAMPLE A}

The starting Hamiltonian for the double-dot system with nine superconducting (SC) leads reads

$$
H=H_{d}+\sum_{j=1}^{9}\left(H_{s, j}+H_{d-s, j}+\sum_{m>j}^{9} H_{s-s, j m}\right)
$$

with the Hamiltonian of the two coupled dots

$$
\begin{aligned}
H_{d}= & \sum_{\alpha=L, R} \sum_{\sigma} \epsilon_{\alpha} d_{\alpha \sigma}^{\dagger} d_{\alpha \sigma} \\
& +\sum_{\sigma} \sigma B_{z}\left(d_{L \sigma}^{\dagger} d_{L \sigma}-d_{R \sigma}^{\dagger} d_{R \sigma}\right) \\
& +v_{0} \sum_{\sigma}\left(d_{L \sigma}^{\dagger} d_{R \sigma}+\text { H.c. }\right)
\end{aligned}
$$

where $B_{z}$ is a local Zeeman field (reversed sign on each dot), $v_{0}$ is the hopping between both dots, $d_{\alpha \sigma}^{\dagger}$ is the creation operator of an electron on the dot $\alpha \in\{L, R\}$ with 
energy $\epsilon_{\alpha}$ and spin $\sigma=\uparrow, \downarrow$. The $j$ th SC lead is described by a BCS Hamiltonian

$$
H_{s, j}=\sum_{\mathbf{k} \sigma} \epsilon_{\mathbf{k}} c_{j \mathbf{k} \sigma}^{\dagger} c_{j \mathbf{k} \sigma}+\sum_{\mathbf{k}}\left(\Delta e^{i \varphi_{j}} c_{j \mathbf{k} \uparrow}^{\dagger} c_{j(-\mathbf{k}) \downarrow}^{\dagger}+\text { H.c. }\right),
$$

where $c_{j \mathbf{k} \sigma}^{\dagger}$ is the creation operator of an electron in the $j$ th lead with momentum $\mathbf{k}$ and spin $\sigma$. Here, $\Delta$ is the SC gap (assumed to be the same for all superconductors) with a phase $\varphi_{j}$ and $\epsilon_{\mathbf{k}}$ is the normal state dispersion in the leads.

To have a correct ordering of the leads, we denote the lead in the top-right corner as $j=1$ and count in the clockwise direction until $j=9$, the lead left to that in the top-right corner (see Fig. 3 in the main text). The phases of the leads read $\varphi_{1}-\pi=\varphi_{5}=\phi_{3}, \varphi_{2}=\varphi_{6}=\phi_{4}, \varphi_{3}=$ $\varphi_{7}-\pi=\phi_{2}$, and $\varphi_{4}=\varphi_{9}=\phi_{0}$. The leads $j=1,2,3,9$ are coupled to the right dot via

$$
H_{d-s, j}=v \sum_{\mathbf{k} \sigma} d_{R \sigma}^{\dagger} c_{j \mathbf{k} \sigma}+\text { H.c., }
$$

while the leads $\tilde{j}=4,5,6,7,8$ are coupled to the left dot via

$$
H_{d-s, \tilde{j}}=v \sum_{\mathbf{k} \sigma} d_{L \sigma}^{\dagger} c_{\tilde{j} \mathbf{k} \sigma}+\text { H.c. }
$$

The leads $\left(j_{0}, m_{0}\right)=(3,4)$ and $\left(j_{0}, m_{0}\right)=(8,9)$ are coupled by a normal hopping

$$
H_{s-s, j_{0} m_{0}}=w_{0} \sum_{\mathbf{k} \sigma} c_{j_{0} \mathbf{k} \sigma}^{\dagger} c_{m_{0} \mathbf{k} \sigma}+\text { H.c., }
$$

whereas the leads $\left(j_{1}, m_{1}\right)=(4,5)$ and $\left(j_{1}, m_{1}\right)=(9,1)$ are connected via spin-orbit coupling $(\mathrm{SOC}) \mathbf{w}_{1}=$ $\left(w_{1 x}, w_{1 y}, w_{1 z}\right)^{\top}$,

$$
H_{s-s, j_{1} m_{1}}=\sum_{\mathbf{k} \sigma} \boldsymbol{c}_{j_{1} \mathbf{k}}^{\dagger}\left(i \mathbf{w}_{1} \cdot \boldsymbol{\sigma} \otimes \tau_{3}\right) \boldsymbol{c}_{m_{1} \mathbf{k}}+\text { H.c. }
$$

with $\boldsymbol{c}_{j \mathbf{k}}^{\dagger}=\left(c_{j \mathbf{k} \uparrow}^{\dagger}, c_{j(-\mathbf{k}) \downarrow}, c_{j \mathbf{k} \downarrow}^{\dagger},-c_{j(-\mathbf{k}) \uparrow}\right)$ the spinor in composed spin and Nambu space. Here, we introduced a set of Pauli matrices $\boldsymbol{\sigma}=\left(\sigma_{1}, \sigma_{2}, \sigma_{3}\right)$ in spin space and another set of Pauli matrices $\tau_{1}, \tau_{2}$, and $\tau_{3}$ in Nambu space.

The leads $\left(j_{2}, m_{2}\right)=(5,6)$ and $\left(j_{2}, m_{2}\right)=(1,2)$ are connected via SOC $\mathbf{w}_{2}=\left(w_{2 x}, w_{2 y}, w_{2 z}\right)^{\top}$,

$$
H_{s-s, j_{2} m_{2}}=\sum_{\mathbf{k} \sigma} \boldsymbol{c}_{j_{2} \mathbf{k}}^{\dagger}\left(i \mathbf{w}_{2} \cdot \boldsymbol{\sigma} \otimes \tau_{3}\right) \boldsymbol{c}_{m_{2} \mathbf{k}}+\text { H.c. }
$$

Similarly, the leads $\left(j_{3}, m_{3}\right)=(6,7)$ and $\left(j_{3}, m_{3}\right)=(2,3)$ are coupled via $\mathrm{SOC} \mathbf{w}_{3}=\left(w_{3 x}, w_{3 y}, w_{3 z}\right)^{\top}$,

$$
H_{s-s, j_{3} m_{3}}=\sum_{\mathbf{k} \sigma} \boldsymbol{c}_{j_{3} \mathbf{k}}^{\dagger}\left(i \mathbf{w}_{3} \cdot \boldsymbol{\sigma} \otimes \tau_{3}\right) \boldsymbol{c}_{m_{3} \mathbf{k}}+\text { H.c. }
$$

Since the hoppings do not depend on quasimomentum $\mathbf{k}$, we can already integrate out $\mathbf{k}$ to obtain effective Green's functions of the SC leads. The Green function of a single $\mathrm{SC}$ lead becomes

$$
g_{s, \varphi_{j}}=-\frac{\pi N_{0}}{\sqrt{\Delta^{2}-\epsilon^{2}}} \sigma_{0} \otimes\left(\epsilon \tau_{0}+\Delta e^{i \varphi_{j} \tau_{3}} \tau_{1}\right),
$$

where $\sigma_{0}$ and $\tau_{0}$ are identities in spin and Nambu spaces, respectively, and $N_{0}$ is the density of states in the normal state. In the low-energy limit $\epsilon \ll \Delta$, we obtain

$$
g_{s, \varphi_{j}} \approx-\pi N_{0}\left(\sigma_{0} \otimes e^{i \varphi_{j} \tau_{3}} \tau_{1}\right) .
$$

For a small hopping and SOC between the leads, $w_{0},\left|\mathbf{w}_{j}\right| \ll v$, we expand the dressed Green function of the two dots $G_{d}$ with the Dyson equation up to first order in the hopping between the leads

$$
G_{d}=g_{d}+g_{d} V_{d s} g_{s}\left(V_{s d}+V_{s s} g_{s} V_{s d}\right) G_{d},
$$

with $g_{d}=\operatorname{diag}\left(g_{d, L}, g_{d, R}\right)$ the bare Green function of the two dots, $g_{s}=\operatorname{diag}\left(g_{s, \varphi_{1}}, \ldots, g_{s, \varphi_{9}}\right)$ the bare Green function of the superconductors,

$$
V_{s s}=\left(\begin{array}{ccccccccc}
0 & W_{2} & 0 & 0 & 0 & 0 & 0 & 0 & W_{1}^{\dagger} \\
W_{2}^{\dagger} & 0 & W_{3} & 0 & 0 & 0 & 0 & 0 & 0 \\
0 & W_{3}^{\dagger} & 0 & W_{0} & 0 & 0 & 0 & 0 & 0 \\
0 & 0 & W_{0} & 0 & W_{1} & 0 & 0 & 0 & 0 \\
0 & 0 & 0 & W_{1}^{\dagger} & 0 & W_{2} & 0 & 0 & 0 \\
0 & 0 & 0 & 0 & W_{2}^{\dagger} & 0 & W_{3} & 0 & 0 \\
0 & 0 & 0 & 0 & 0 & W_{3}^{\dagger} & 0 & 0 & 0 \\
0 & 0 & 0 & 0 & 0 & 0 & 0 & 0 & W_{0} \\
W_{1} & 0 & 0 & 0 & 0 & 0 & 0 & W_{0} & 0
\end{array}\right)
$$

the interaction between the superconductors, and

$$
\begin{aligned}
V_{d s} & =\left(\begin{array}{ccccccccc}
0 & 0 & 0 & v_{s d} & v_{s d} & v_{s d} & v_{s d} & v_{s d} & 0 \\
v_{s d} & v_{s d} & v_{s d} & 0 & 0 & 0 & 0 & 0 & v_{s d}
\end{array}\right) \\
& =V_{s d}^{\dagger}
\end{aligned}
$$

the interaction between dots and superconductors, whereas $v_{s d}=v\left(\sigma_{0} \otimes \tau_{3}\right), W_{j}=i\left(\mathbf{w}_{j} \cdot \boldsymbol{\sigma} \otimes \tau_{3}\right)$, and $W_{0}=w_{0}\left(\sigma_{0} \otimes\right.$ $\left.\tau_{3}\right)$. From this, we obtain the effective Hamiltonian of the double-dot system as $H_{\text {eff }}=H_{d}+\Sigma_{0}+\Sigma_{1}$ with the zeroth-order contribution

$$
\begin{aligned}
\Sigma_{0}= & \frac{1}{2}\left(\mathbf{d}_{L}^{\dagger}, \mathbf{d}_{R}^{\dagger}\right) V_{d s} g_{s} V_{s d}\left(\begin{array}{c}
\mathbf{d}_{L} \\
\mathbf{d}_{R}
\end{array}\right) \\
= & \Gamma\left(\sum_{j=0,1,3,4} e^{i \phi_{j}}-e^{i \phi_{2}}\right) d_{L \uparrow}^{\dagger} d_{L \downarrow}^{\dagger}+\text { H.c. } \\
& +\Gamma\left(\sum_{j=0,2,4} e^{i \phi_{j}}-e^{i \phi_{3}}\right) d_{R \uparrow}^{\dagger} d_{R \downarrow}^{\dagger}+\text { H.c., }
\end{aligned}
$$


where $\Gamma=\pi N_{0} v^{2}$ and the spinor $\mathbf{d}_{\alpha}^{\dagger}=\left(d_{\alpha \uparrow}^{\dagger}, d_{\alpha \downarrow}, d_{\alpha \downarrow}^{\dagger},-d_{\alpha \uparrow}\right)$, and the first-order contribution

$$
\begin{aligned}
\Sigma_{1}= & \frac{1}{2}\left(\mathbf{d}_{L}^{\dagger}, \mathbf{d}_{R}^{\dagger}\right) V_{d s} g_{s} V_{s s} g_{s} V_{s d}\left(\begin{array}{c}
\mathbf{d}_{L} \\
\mathbf{d}_{R}
\end{array}\right) \\
= & -\epsilon_{T}\left(e^{i \phi_{1}}+e^{-i \phi_{2}}\right)\left(d_{L \uparrow}^{\dagger} d_{R \uparrow}+d_{L \downarrow}^{\dagger} d_{R \downarrow}\right)+\text { H.c. } \\
& -\frac{2 \epsilon_{T}}{w_{0}}\left[w_{1 z} \sin \left(\phi_{3}\right)+w_{2 z} \sin \left(\phi_{4}-\phi_{3}\right)+w_{3 z} \sin \left(\phi_{4}-\phi_{2}\right)\right] \sum_{\sigma} \sigma\left(d_{L \sigma}^{\dagger} d_{L \sigma}-d_{R \sigma}^{\dagger} d_{R \sigma}\right) \\
& -\frac{2 \epsilon_{T}}{w_{0}}\left[\left(w_{1 x}-i w_{1 y}\right) \sin \left(\phi_{3}\right)+\left(w_{2 x}-i w_{2 y}\right) \sin \left(\phi_{4}-\phi_{3}\right)+\left(w_{3 x}-i w_{3 y}\right) \sin \left(\phi_{4}-\phi_{2}\right)\right] \\
& \times\left(d_{L \uparrow}^{\dagger} d_{L \downarrow}-d_{R \uparrow}^{\dagger} d_{R \downarrow}\right)+\text { H.c., }
\end{aligned}
$$

where we have defined $\epsilon_{T}=\pi^{2} N_{0}^{2} v^{2} w_{0}$. From Eq. (A15) we see that the coupling between the superconductors and the dots generates an effective Cooper pairing with gap $\Gamma$ on the double-dot system that we suppress via strong Coulomb interactions described by the Hamiltonian

$$
H_{C}=U\left(n_{L \uparrow} n_{L \downarrow}+n_{R \uparrow} n_{R \downarrow}\right) .
$$

We determine the Hamiltonian $H_{1 e}$ in the odd-parity regime in the limit $U \rightarrow \infty$. As states involving three electrons on the two dots are now forbidden, i.e., the injection of Cooper pairs in $\Sigma_{0}$ is suppressed, the Hamiltonian simply becomes $H_{1 e}=H_{d}+\Sigma_{1}$, which takes the form presented in Eq. (1) in the main text, with the functions $Z(\lambda), K(\lambda)$, and $F(\lambda)$ as provided in Eqs. (2) in the main text.

\section{APPENDIX B. STABILITY OF THE TOPOLOGICAL PHASE IN EXAMPLE A}

In Example $\mathrm{A}$, there can only exist a topological phase for $0<\left|v_{0}\right|<2\left|\epsilon_{T}\right|$ since, for $\left|v_{0}\right|>2\left|\epsilon_{T}\right|$, we have $|K(\lambda)|>0$ and the gap between the two states $E_{\text {gap }}=$ $2 \sqrt{\left|B_{z}+Z\right|^{2}+|K|^{2}+|F|^{2}}$ cannot be closed, always leaving the system in the trivial state; see Fig. 7. In the range $0<\left|v_{0}\right|<2\left|\epsilon_{T}\right|$, the topological phase also exists for a wide region of $B_{z}$. For instance, even for a small $v_{0}$, a relatively large region in $B_{z}$ is topological. In fact, $v_{0}$ changes primarily the size of the region where the second Chern number is 2 (see the main text for the definition of the second Chern number), as depicted in Fig. 7(b).

The size of the topological region in $B_{z}$ is mainly influenced by the magnitude of the SOC, as shown in Fig. 8(a). Another important fact is that if the set $\mathbf{w}_{1}, \mathbf{w}_{2}$, and $\mathbf{w}_{3}$ is linearly dependent, there is no topological phase at all. In Fig. 8(b) we define the SOCs as $\mathbf{w}_{1} / w_{0}=(0.5,0.5 g, 0.5 g)$, $\mathbf{w}_{2} / w_{0}=(0.5 g, 0.5,0.5 g)$, and $\mathbf{w}_{3} / w_{0}=(0.5 g, 0.5 g, 0.5)$ such that, for $g=0$, they are pairwise orthogonal and, for $g=1$, they are equal, $\mathbf{w}_{1}=\mathbf{w}_{2}=\mathbf{w}_{3}$. This shows that, for the orthogonal case $g=0$, the topological region in $B_{z}$ is largest with decreasing size as $g$ approaches $g=1$, for which the topological region vanishes completely.

\section{APPENDIX C. DERIVATION OF EXAMPLE B}

The starting Hamiltonian for the double-dot system with four superconducting leads and four superconducting leads with a local exchange field reads

$$
H=H_{d}+\sum_{j=1}^{8}\left(H_{s, j}+H_{d-s, j}+\sum_{m>j} H_{s-s, j m}\right),
$$

where the Hamiltonian of the dots $H_{d}$ with a local magnetic field $B_{z}$ is

$$
H_{d}=\sum_{\alpha=L, R} \sum_{\sigma} \epsilon_{\alpha} d_{\alpha \sigma}^{\dagger} d_{\alpha \sigma}+\sum_{\sigma} \sigma B_{z}\left(d_{L \sigma}^{\dagger} d_{L \sigma}-d_{R \sigma}^{\dagger} d_{R \sigma}\right)
$$

The variables are the same as explained above; see Eq. (A2). However, note that the two dots are not directly coupled in comparison to Example A. To have a correct ordering of the leads, we denote the superconducting lead in the top-left corner as $j=1$ and count them in a clockwise direction until the lead in the bottom-left corner $(j=8)$; see Fig. 3 in the main text. Hence, the superconducting leads $j=1,2,3,4$ do not have a local exchange field and are described by a BCS Hamiltonian

$$
H_{s, j}=\sum_{\mathbf{k}, \sigma} \epsilon_{\mathbf{k}} c_{j \mathbf{k} \sigma}^{\dagger} c_{j \mathbf{k} \sigma}+\sum_{\mathbf{k}} \Delta\left(e^{i \varphi_{j}} c_{j \mathbf{k} \uparrow}^{\dagger} c_{j(-\mathbf{k}) \downarrow}^{\dagger}+\text { H.c. }\right)
$$

The leads $\tilde{j}=5,6,7,8$ have a local exchange field $h$ with magnetization direction $\tilde{\theta}_{\tilde{j}}$ (in reference to the magnetic field direction on the dots) described by the Hamiltonian 

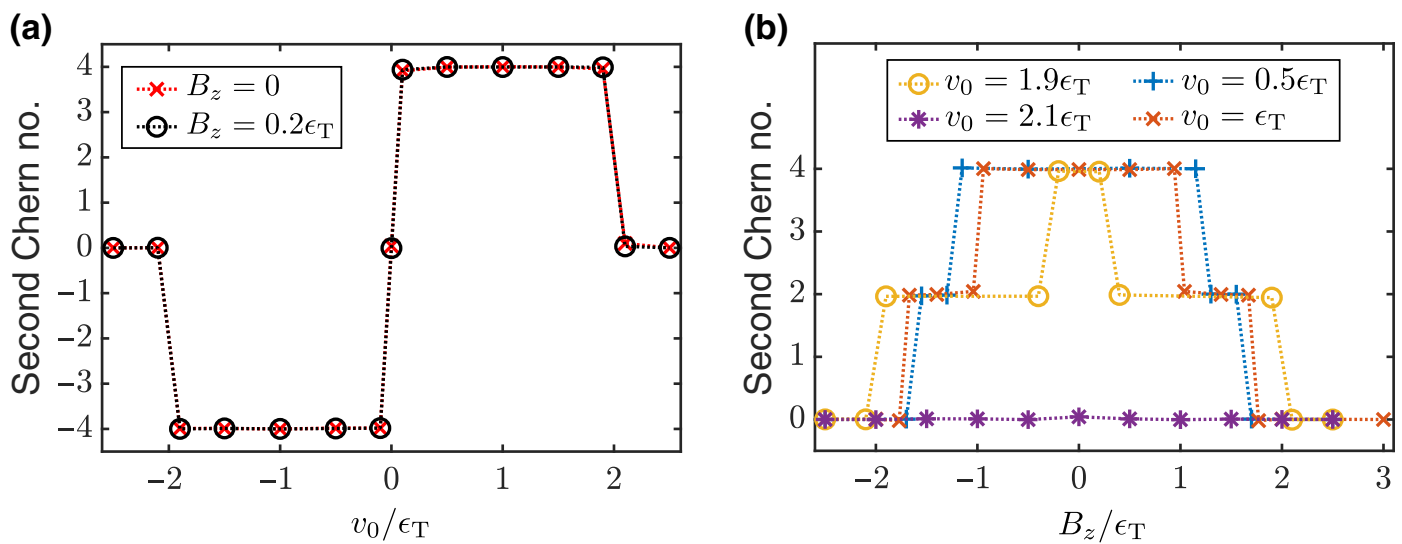

FIG. 7. (a) Second Chern number for Example A for $\mathbf{w}_{1} / w_{0}=(-0.5,0,0.5), \mathbf{w}_{2} / w_{0}=(0,0.5,1)$, and $\mathbf{w}_{3} / w_{0}=(0.5,0,0.5)$ as a function of the direct coupling between the dots $v_{0}$ for different magnetic fields $B_{z}$. (b) Second Chern number for Example A as a function of the magnetic field $B_{z}$ for different couplings $v_{0}$. All results are evaluated numerically with Monte Carlo integration for a maximum of $N=10^{8}$ integration points.

$$
\begin{aligned}
H_{s, \tilde{j}}= & \sum_{\mathbf{k}, \sigma}\left[\epsilon_{\mathbf{k}}+\sigma h \cos \left(\tilde{\theta}_{\tilde{j}}\right)\right] c_{\tilde{j} \mathbf{k} \sigma}^{\dagger} c_{\tilde{j} \mathbf{k} \sigma} \\
& +\sum_{\mathbf{k}} h \sin \left(\tilde{\theta}_{\tilde{j}}\right)\left(c_{\tilde{j} \mathbf{k} \uparrow}^{\dagger} c_{\tilde{j} \mathbf{k} \downarrow}+\text { H.c. }\right) \\
& +\sum_{\mathbf{k}} \Delta\left(e^{i \varphi_{\tilde{j}}} c_{\tilde{j} \mathbf{k} \uparrow}^{\dagger} c_{\tilde{j}(-\mathbf{k}) \downarrow}^{\dagger}+\text { H.c. }\right)
\end{aligned}
$$

The phases of the leads are (as depicted in Fig. 3 in the main text) $\varphi_{1}=\varphi_{3}=\varphi_{5}=\varphi_{7}=\phi_{0}, \varphi_{2}=\varphi_{4}-\pi=\phi_{1}$, and $\tilde{\varphi}_{6}=\tilde{\varphi}_{8}=\phi_{2}$. The magnetization directions are $\tilde{\theta}_{8}=$ $\tilde{\theta}_{5}-\pi=\theta_{1}$ and $\tilde{\theta}_{7}=\tilde{\theta}_{6}-\pi=\theta_{2}$.

The leads (without an exchange field) $j_{L}=1,2$ are coupled to the left dot, whereas the leads (without an exchange field) $j_{R}=3,4$ are coupled to the right dot. With an exchange field, the leads $\tilde{j}_{L}=7,8$ are coupled to the left dot and the leads $\tilde{j}_{R}=5,6$ are coupled to the right dot:

$$
\begin{aligned}
& H_{d-s, j_{L}}=v \sum_{\mathbf{k}, \sigma}\left(d_{L \sigma}^{\dagger} c_{j_{L} \mathbf{k} \sigma}+\text { H.c. }\right), \\
& H_{d-s, j_{R}}=v \sum_{\mathbf{k}, \sigma}\left(d_{R \sigma}^{\dagger} c_{j_{R} \mathbf{k} \sigma}+\text { H.c. }\right),
\end{aligned}
$$

(b)

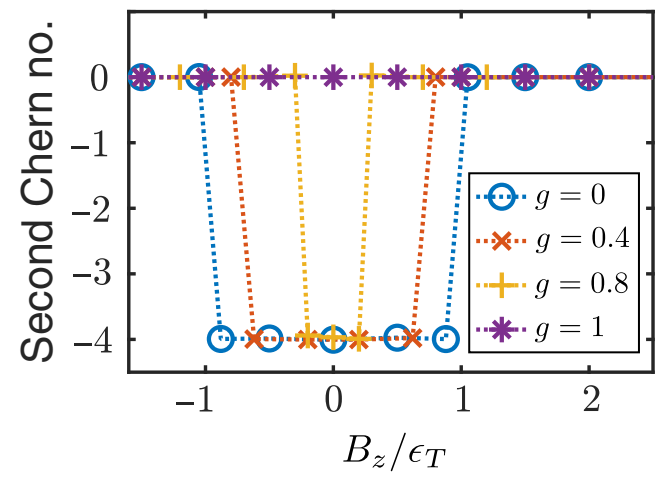

FIG. 8. (a) Second Chern number for Example A for $\mathbf{w}_{1} / w_{0} \propto(1,0,0), \mathbf{w}_{2} / w_{0} \propto(0,1,0)$, and $\mathbf{w}_{3} / w_{0} \propto(0,0,1)$ as a function of the magnetic field $B_{z}$ for different absolute magnitudes of the SOC with $v_{0}=\epsilon_{T}$. (b) Second Chern number for Example A as a function of the magnetic field $B_{z}$ with $v_{0}=0.1 \epsilon_{T}, \mathbf{w}_{1} / w_{0}=(0.5,0.5 g, 0.5 g), \mathbf{w}_{2} / w_{0}=(0.5 g, 0.5,0.5 g)$, and $\mathbf{w}_{3} / w_{0}=(0.5 g, 0.5 g, 0.5)$, such that, for $g=0$, the SOCs are pairwise orthogonal and, for $g=1$, they are equal. All results are evaluated numerically with Monte Carlo integration for a maximum of $N=10^{8}$ integration points. 
The leads (without an exchange field) $j=2$ and $m=3$ are coupled by

$$
H_{s-s, 23}=w_{0} \sum_{\mathbf{k}, \sigma}\left(c_{2 \mathbf{k} \sigma}^{\dagger} c_{3 \mathbf{k} \sigma}+\text { H.c. }\right),
$$

where $w_{0}$ is the normal hopping energy.

Similarly, the leads with an exchange field, $j=5$ and $m=6$, are coupled by

$$
H_{S-s, 56}=\tilde{w}_{0} \sum_{\mathbf{k}, \sigma}\left(c_{5 \mathbf{k} \sigma}^{\dagger} c_{6 \mathbf{k} \sigma}+\text { H.c. }\right),
$$

where $\tilde{w}_{0}$ is the normal hopping energy. The couplings between the leads $(j, m)=(5,6)$ and $(j, m)=(7,8)$ are

$$
H_{s-s, j m}=\tilde{w}_{0} e^{i \pi / 2} \sum_{\mathbf{k}, \sigma} c_{j \mathbf{k} \sigma}^{\dagger} c_{m \mathbf{k} \sigma}+\text { H.c., }
$$

where we assumed that there is a phase shift. The leads $(j, m)=(1,2)$ and $(j, m)=(3,4)$ are connected via the spin-dependent hopping

$$
H_{S-S, j s, m_{s}}=w_{0} e^{i \pi / 2} \sum_{\mathbf{k}, \sigma} \sigma c_{j \mathbf{k} \sigma}^{\dagger} c_{m \mathbf{k} \sigma}+\text { H.c. }
$$

Since the hoppings do not depend on quasimomentum $\mathbf{k}$, we can already integrate out $\mathbf{k}$ to obtain effective Green's functions of the SC leads. The Green function of a single superconducting lead with phase $\varphi_{j}$, a superconducting gap $\Delta$, a local exchange field $h$, and a magnetization angle $\tilde{\theta}_{j}$ (with respect to the magnetization direction on the dots) becomes, in the limit of low energy $\epsilon \ll h, \Delta$,

$$
\begin{aligned}
g_{s, \varphi_{j}, \tilde{\theta}_{j}}= & \frac{-\pi N_{0}}{\sqrt{\Delta^{2}-h^{2}}}\left[-h \cos \left(\tilde{\theta}_{j}\right)\left(\sigma_{3} \otimes \tau_{0}\right)\right] \\
& +\frac{\pi N_{0}}{\sqrt{\Delta^{2}-h^{2}}}\left[h \sin \left(\tilde{\theta}_{j}\right)\left(\sigma_{1} \otimes \tau_{0}\right)\right. \\
& \left.-\Delta\left(\sigma_{0} \otimes e^{i \phi_{j} \tau_{3}} \tau_{1}\right)\right] .
\end{aligned}
$$

Again, for small hopping between the leads $w_{0} \ll v$ and $\tilde{w}_{0} \ll \tilde{v}$, we expand the dressed Green function of the double-dot system $G_{d}$ with the Dyson equation up to first order in the hopping between the leads. This yields

$$
G_{d}=g_{d}+g_{d} V_{d s} g_{s}\left(V_{s d}+V_{s s} g_{s} V_{s d}\right) g_{d}
$$

with $g_{d}=\operatorname{diag}\left(g_{d, L}, g_{d, R}\right)$ the unperturbed Green function of the two dots, $g_{s}=\operatorname{diag}\left(g_{s, \varphi_{1}}, \ldots, g_{s, \varphi_{4}}, g_{s, \varphi_{5}, \theta_{5}}, \ldots\right.$, $\left.g_{s, \varphi_{8}, \theta_{8}}\right)$ the Green function of the superconductors,

$$
V_{s s}=\left(\begin{array}{cccccccc}
0 & W_{\sigma} & 0 & 0 & 0 & 0 & 0 & 0 \\
W_{\sigma}^{\dagger} & 0 & W_{0} & 0 & 0 & 0 & 0 & 0 \\
0 & W_{0} & 0 & W_{\sigma} & 0 & 0 & 0 & 0 \\
0 & 0 & W_{\sigma}^{\dagger} & 0 & 0 & 0 & 0 & 0 \\
0 & 0 & 0 & 0 & 0 & \tilde{W}_{\pi / 2} & 0 & 0 \\
0 & 0 & 0 & 0 & \tilde{W}_{\pi / 2}^{\dagger} & 0 & \tilde{W}_{0} & 0 \\
0 & 0 & 0 & 0 & 0 & \tilde{W}_{0} & 0 & \tilde{W}_{\pi / 2} \\
0 & 0 & 0 & 0 & 0 & 0 & \tilde{W}_{\pi / 2} & 0
\end{array}\right)
$$

the interaction between the superconductors, and

$V_{d s}=\left(\begin{array}{cccccccc}v_{s d} & v_{s d} & 0 & 0 & 0 & 0 & \tilde{v}_{s d} & \tilde{v}_{s d} \\ 0 & 0 & v_{s d} & v_{s d} & \tilde{v}_{s d} & \tilde{v}_{s d} & 0 & 0\end{array}\right)=V_{s d}^{\dagger}$

the interaction between the dots and the superconductors. Here, $\tilde{v}_{s d}=\tilde{v}\left(\sigma_{0} \otimes \tau_{3}\right)$ is the normal hopping between dots and leads with an exchange field, the normal complex hopping $\tilde{W}_{\pi / 2}=\tilde{w}_{0} \sigma_{0} \otimes\left(e^{i \pi \tau_{3} / 2} \tau_{3}\right)$, and the complex spin-dependent hopping $W_{\sigma}=w_{0} \sigma_{3} \otimes\left(e^{i \pi \tau_{3} / 2} \tau_{3}\right)$.

From this, we obtain the effective Hamiltonian of the double-dot system as $H_{\text {eff }}=H_{d}+\Sigma_{0}+\Sigma_{1}$, with the zeroth-order contribution

$$
\Sigma_{0}=\frac{1}{2}\left(\mathbf{d}_{L}^{\dagger}, \mathbf{d}_{R}^{\dagger}\right) V_{d s} g_{s} V_{s d}\left(\begin{array}{l}
\mathbf{d}_{L} \\
\mathbf{d}_{R}
\end{array}\right)=\Sigma_{0}^{(1)}+\Sigma_{0}^{(2)}
$$

$$
\begin{aligned}
\Sigma_{0}^{(1)}= & {\left[\Gamma\left(e^{i \phi_{0}}+e^{i \phi_{1}}\right)+\frac{\epsilon_{h} \Delta}{\sqrt{\Delta^{2}-h^{2}}}\left(e^{i \phi_{0}}+e^{i \phi_{2}}\right)\right] d_{L \uparrow}^{\dagger} d_{L \downarrow}^{\dagger} } \\
& + \text { H.c. }+\left[\Gamma\left(e^{i \phi_{0}}-e^{i \phi_{1}}\right)+\frac{\epsilon_{h} \Delta}{\sqrt{\Delta^{2}-h^{2}}}\left(e^{i \phi_{0}}+e^{i \phi_{2}}\right)\right] \\
& d_{R \uparrow}^{\dagger} d_{R \downarrow}^{\dagger}+\text { H.c. }, \quad(\mathrm{C} 17 \mathrm{~b})
\end{aligned}
$$




$$
\begin{aligned}
\Sigma_{0}^{(2)}= & \frac{\epsilon_{h} h}{\sqrt{\Delta^{2}-h^{2}}}\left[\cos \left(\theta_{1}\right)+\cos \left(\theta_{2}\right)\right] \\
& \sum_{\sigma} \sigma\left(d_{L \sigma}^{\dagger} d_{L \sigma}-d_{R \sigma}^{\dagger} d_{R \sigma}\right)+\frac{\epsilon_{h} h}{\sqrt{\Delta^{2}-h^{2}}} \\
& \times\left[\sin \left(\theta_{1}\right)+\sin \left(\theta_{2}\right)\right]\left(d_{L \uparrow}^{\dagger} d_{L \downarrow}-d_{R \uparrow}^{\dagger} d_{R \downarrow}\right)+\text { H.c. }
\end{aligned}
$$

where $\Gamma=\pi N_{0} v^{2}$ and $\epsilon_{h}=\pi N_{0} \tilde{v}^{2}$, and the first-order contribution

$$
\Sigma_{1}=\frac{1}{2}\left(\mathbf{d}_{L}^{\dagger}, \mathbf{d}_{R}^{\dagger}\right) V_{d s} g_{s} V_{s s} g_{s} V_{s d}\left(\begin{array}{c}
\mathbf{d}_{L} \\
\mathbf{d}_{R}
\end{array}\right)=\Sigma_{1}^{(1)}+\Sigma_{1}^{(2)}
$$

$$
\begin{aligned}
\Sigma_{1}^{(1)}= & 2 \epsilon_{T} \cos \left(\phi_{0}-\phi_{1}-\frac{\pi}{2}\right) \sum_{\sigma} \sigma\left(d_{R \sigma}^{\dagger} d_{R \sigma}-d_{L \sigma}^{\dagger} d_{L \sigma}\right) \\
& -\frac{2 \tilde{\epsilon}_{T} \Delta^{2}}{\Delta^{2}-h^{2}} \cos \left(\phi_{2}-\phi_{0}-\frac{\pi}{2}\right) \sum_{\alpha, \sigma} d_{\alpha \sigma}^{\dagger} d_{\alpha \sigma} \\
& +\frac{2 \tilde{\epsilon}_{T} h^{2}}{\Delta^{2}-h^{2}} \sin \left(\theta_{2}-\theta_{1}\right)\left(i d_{L \uparrow}^{\dagger} d_{L \downarrow}-i d_{R \uparrow}^{\dagger} d_{R \downarrow}+\text { H.c. }\right) \\
& -\left(\frac{\tilde{\epsilon}_{T} h^{2}}{\Delta^{2}-h^{2}}+\epsilon_{T} e^{i\left(\phi_{1}-\phi_{0}\right)}+\frac{\Delta^{2} \tilde{\epsilon}_{T}}{\Delta^{2}-h^{2}} e^{i\left(\phi_{0}-\phi_{2}\right)}\right) \\
& \left(\sum_{\sigma} d_{L \sigma}^{\dagger} d_{R \sigma}\right)+\text { H.c., }
\end{aligned}
$$

$$
\begin{aligned}
\Sigma_{1}^{(2)}= & \tilde{\epsilon}_{T} \frac{\Delta h \sin \left(\theta_{2}\right)}{\Delta^{2}-h^{2}}\left(e^{i \phi_{2}}-e^{i \phi_{0}}\right)\left(d_{L \uparrow}^{\dagger} d_{R \uparrow}^{\dagger}+d_{L \downarrow}^{\dagger} d_{R \downarrow}^{\dagger}\right)+\text { H.c. } \\
& +\tilde{\epsilon}_{T} \frac{\Delta h \cos \left(\theta_{2}\right)}{\Delta^{2}-h^{2}}\left(e^{i \phi_{2}}-e^{i \phi_{0}}\right)\left(d_{L \uparrow}^{\dagger} d_{R \downarrow}^{\dagger}+d_{L \downarrow}^{\dagger} d_{R \uparrow}^{\dagger}\right) \\
& + \text { H.c., }
\end{aligned}
$$

where $\epsilon_{T}=\pi^{2} N_{0}^{2} v^{2} w_{0}$ and $\tilde{\epsilon}_{T}=\pi^{2} N_{0}^{2} \tilde{v}^{2} \tilde{w}_{0}$. From Eqs. (C17) and (C18) we see that effective local Cooper pairing terms on the double-dot system are proximity generated, which we suppress by considering strong Coulomb interactions described by the Hamiltonian

$$
H_{C}=U\left(n_{L \uparrow} n_{L \downarrow}+n_{R \uparrow} n_{R \downarrow}\right) .
$$

We determine the Hamiltonian $H_{1 e}$ in the odd-parity regime in the limit $U \rightarrow \infty$. As states involving three electrons on the two dots are now forbidden, i.e., the injection of Cooper pairs in $\Sigma_{0}^{(1)}+\Sigma_{1}^{(2)}$ is suppressed, the Hamiltonian simply becomes $H_{1 e}=H_{d}+\Sigma_{0}^{(2)}+\Sigma_{1}^{(1)}$, which takes the form presented in Eq. (1) in the main text, with the functions $Z(\lambda), K(\lambda)$, and $F(\lambda)$ as provided in Eqs. (3) in the main text.

\section{APPENDIX D. STABILITY OF THE TOPOLOGICAL PHASE IN EXAMPLE B}

As depicted in Fig. 9(a), there exists a topological phase for approximately $0.6 \lesssim \tilde{\epsilon}_{T} / \epsilon_{T} \lesssim 1$ even within the range $-2 \epsilon_{T} \lesssim B_{z} \lesssim-\epsilon_{T}$. For a similar topological region in $B_{z}$, the ratio $\epsilon_{h} / \epsilon_{T}$ takes values $1 \lesssim \epsilon_{h} / \epsilon_{T} \lesssim 2.5$ and decreases in $B_{z}$ for $\epsilon_{h} / \epsilon_{T}<1$ and $\epsilon_{h} / \epsilon_{T}>2.5$; see Fig. 9(b). In Fig. 10 the topological region in dependence of $h / \Delta$ is shown. For small exchange fields $h$ in the leads, there is no topological phase. However, for $0.4<h / \Delta<0.9$, a topological region appears. For $0.6<h / \Delta<0.8$, the nontrivial region is the largest in $B_{z}$. The topological phase vanishes for $0.4<h / \Delta<0.6$ for large values of $B_{z}=$ $-3.5 \epsilon_{T}$, whereas for $0.8<h / \Delta<0.9$, the region vanishes for small magnitudes of the local magnetic field $B_{z}=$ $-\epsilon_{T}$. Hence, for all parameters, the topological region appears within reasonable boundaries. Only for the ratio $\tilde{\epsilon}_{T} / \epsilon_{T}$ there exists a small region resulting in a topological phase. Since $\tilde{\epsilon}_{T}$ and $\epsilon_{T}$ are the characteristic energies for

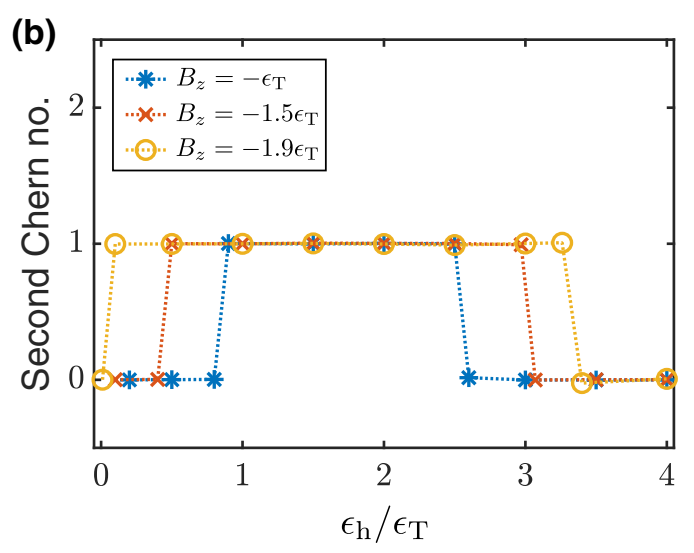

FIG. 9. Second Chern number for Example B for $h / \Delta=0.5$ as functions of (a) $\tilde{\epsilon}_{T} / \epsilon_{T}$ with $\epsilon_{h}=\epsilon_{T}$ and (b) $\epsilon_{h} / \epsilon_{T}$ with $\epsilon_{T}=4 \tilde{\epsilon}_{T} / 3$ for different magnetic fields $B_{z}$. All results are evaluated numerically with Monte Carlo integration for a maximum of $N=10^{8}$ integration points. 


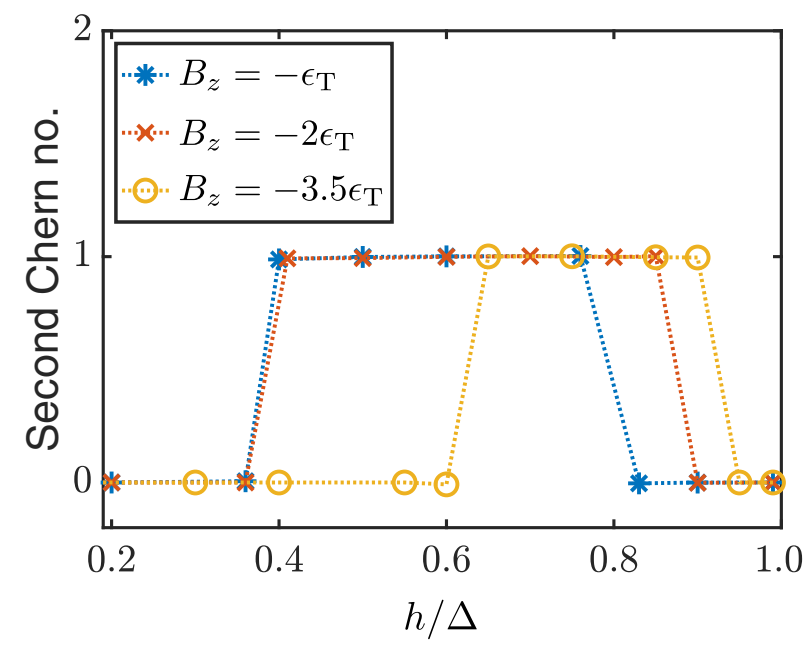

FIG. 10. Second Chern number in Example B for $\epsilon_{T}=\epsilon_{h}=$ $4 \tilde{\epsilon}_{T} / 3$ as a function of the ratio $h / \Delta$ for different magnetic fields $B_{z}$. All results are evaluated numerically with Monte Carlo integration for a maximum of $N=10^{8}$ integration points.

the same hopping process only with and without a local exchange field on the SC leads, the range $\tilde{\epsilon}_{T} \lesssim \epsilon_{T}$ should be experimentally accessible.

\section{APPENDIX E. NON-ABELIAN BERRY ROTATIONS IN EXAMPLE B}

In Example $\mathrm{B}$ the rotation around the polar angle $\theta$ between the degenerate states $|E\rangle_{-}^{(1)}$ and $\left|E_{-}^{(2)}\right\rangle$ can be achieved by varying $\theta_{1}$ and $\theta_{2}$ along the cyclic path defined by $\theta_{1}=\theta_{2}=0 \rightarrow 2 \pi$ and by setting the SC phases to $\phi_{1}=\phi_{2}=0$. The rotation around the azimuthal angle $\varphi$

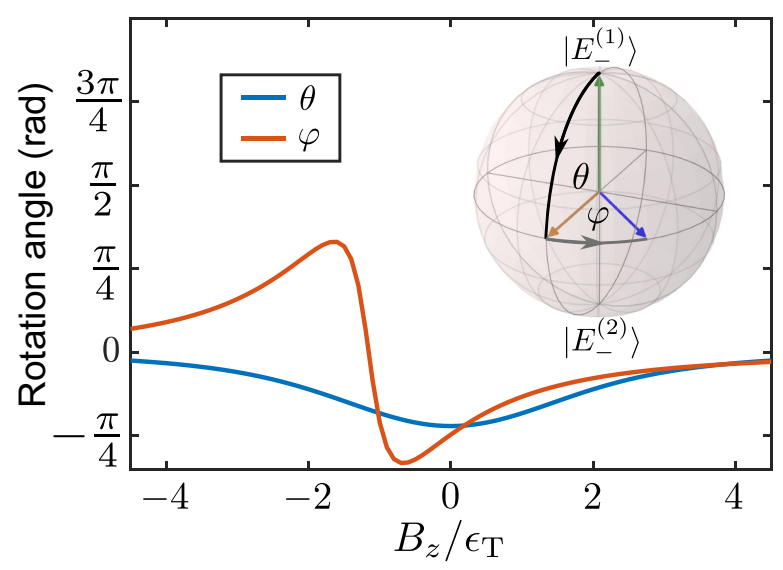

FIG. 11. Bloch sphere of the degenerate subspace $E_{-}$with the possible rotations around the polar angle $\theta$ and the azimuthal angle $\varphi$ for Example B (top-right corner). Numerically determined angles of the Berry rotations $\varphi$ (by changing adiabatically $\phi_{2}: 0 \rightarrow 2 \pi$ ) and $\theta$ (by changing adiabatically $\theta_{1}, \theta_{2}: 0 \rightarrow 2 \pi$ ) as a function of the magnetic field $B_{z}$ for $\epsilon_{T}=\epsilon_{h}=4 \tilde{\epsilon}_{T} / 3$ and $h / \Delta=0.5$. is implemented by the adiabatic change $\phi_{2}=0 \rightarrow 2 \pi$ and by simply setting $\phi_{1}=\theta_{1}=\theta_{2}=0$. The rotation angles depend on the magnetic field $B_{z}$, as presented in Fig. 11 . For the concrete set of parameters of Fig. 11 (parameters are set equal to those in Fig. 4 of the main text), the rotation for a single cyclic evolution is restricted to $0.3 \lesssim \varphi / \pi \lesssim$ -0.3 for the azimuthal rotation and $0 \lesssim \theta / \pi \lesssim-0.2$ for the polar rotation. Hence, a single cyclic path is not enough to rotate for larger angles of $\theta$ and $\varphi$. However, one can repeat these cyclic paths, resulting in integer multiples of the rotation angles of a single cyclic evolution.

[1] K. von Klitzing, G. Dorda, and M. Pepper, New Method for High-Accuracy Determination of the Fine-Structure Constant Based on Quantized Hall Resistance, Phys. Rev. Lett. 45, 494 (1980).

[2] D. J. Thouless, M. Kohmoto, M. P. Nightingale, and M. den Nijs, Quantized Hall Conductance in a Two-Dimensional Periodic Potential, Phys. Rev. Lett. 49, 405 (1982).

[3] Y. Hatsugai, Chern Number and Edge States in the Integer Quantum Hall Effect, Phys. Rev. Lett. 71, 3697 (1993).

[4] M. Z. Hasan and C. L. Kane, Colloquium: Topological insulators, Rev. Mod. Phys. 82, 3045 (2010).

[5] M. Sato and Y. Ando, Topological superconductors: A review, Rep. Prog. Phys. 80, 076501 (2017).

[6] S. D. Sarma, M. Freedman, and C. Nayak, Majorana zero modes and topological quantum computation, npj Quantum Inf. 1, 15001 (2015).

[7] M. T. Deng, S. Vaitiekènas, E. B. Hansen, J. Danon, M. Leijnse, K. Flensberg, J. Nygård, P. Krogstrup, and C. M. Marcus, Majorana bound state in a coupled quantum-dot hybrid-nanowire system, Science 354, 1557 (2016).

[8] D. Aasen, M. Hell, R. V. Mishmash, A. Higginbotham, J. Danon, M. Leijnse, T. S. Jespersen, J. A. Folk, C. M. Marcus, K. Flensberg, et al., Milestones toward MajoranaBased Quantum Computing, Phys. Rev. X 6, 031016 (2016).

[9] T. Karzig, C. Knapp, R. M. Lutchyn, P. Bonderson, M. B. Hastings, C. Nayak, J. Alicea, K. Flensberg, S. Plugge, Y. Oreg, et al., Scalable designs for quasiparticle-poisoningprotected topological quantum computation with Majorana zero modes, Phys. Rev. B 95, 235305 (2017).

[10] F. Wilczek and A. Zee, Appearance of Gauge Structure in Simple Dynamical Systems, Phys. Rev. Lett. 52, 2111 (1984).

[11] J. Pachos, P. Zanardi, and M. Rasetti, Non-Abelian Berry connections for quantum computation, Phys. Rev. A 61, 010305(R) (1999).

[12] P. Zanardi and M. Rasetti, Holonomic quantum computation, Phys. Lett. A 264, 94 (1999).

[13] T. Albash and D. A. Lidar, Adiabatic quantum computation, Rev. Mod. Phys. 90, 015002 (2018).

[14] R.-P. Riwar, M. Houzet, J. S. Meyer, and Y. V. Nazarov, Multi-terminal Josephson junctions as topological matter, Nat. Commun. 7, 11167 (2016).

[15] E. Eriksson, R.-P. Riwar, M. Houzet, J. S. Meyer, and Y. V. Nazarov, Topological transconductance quantization in a 
four-terminal Josephson junction, Phys. Rev. B 95, 075417 (2017).

[16] H.-Y. Xie, M. G. Vavilov, and A. Levchenko, Topological Andreev bands in three-terminal Josephson junctions, Phys. Rev. B 96, 161406(R) (2017).

[17] J. S. Meyer and M. Houzet, Nontrivial Chern Numbers in Three-Terminal Josephson Junctions, Phys. Rev. Lett. 119, 136807 (2017).

[18] H.-Y. Xie, M. G. Vavilov, and A. Levchenko, Weyl nodes in Andreev spectra of multiterminal Josephson junctions: Chern numbers, conductances, and supercurrents, Phys. Rev. B 97, 035443 (2018).

[19] O. Deb, K. Sengupta, and D. Sen, Josephson junctions of multiple superconducting wires, Phys. Rev. B 97, 174518 (2018).

[20] R. L. Klees, G. Rastelli, J. C. Cuevas, and W. Belzig, Microwave Spectroscopy Reveals the Quantum Geometric Tensor of Topological Josephson Matter, Phys. Rev. Lett. 124, 197002 (2020).

[21] H.-Y. Xie and A. Levchenko, Topological supercurrents interaction and fluctuations in the multiterminal Josephson effect, Phys. Rev. B 99, 094519 (2019).

[22] A. Blais, S. M. Girvin, and W. D. Oliver, Quantum information processing and quantum optics with circuit quantum electrodynamics, Nat. Phys. 16, 247 (2020).

[23] J. M. Martinis, M. H. Devoret, and J. Clarke, Quantum Josephson junction circuits and the dawn of artificial atoms, Nat. Phys. 16, 234 (2020).

[24] B. Josephson, Possible new effect in superconducting tunneling, Phys. Lett. 1, 251 (1962).

[25] A. A. Golubov, Y. M. Kupriyanov, and E. Il'chev, The current-phase relation in Josephson junctions, Rev. Mod. Phys. 76, 411 (2004).

[26] L. Glazman and K. Matveev, Resonant Josephson current through Kondo impurities in a tunnel barrier, JETP Lett. 49, 659 (1989).

[27] M. R. Buitelaar, T. Nussbaumer, and C. Schönenberger, Quantum Dot in the Kondo Regime Coupled to Superconductors, Phys. Rev. Lett. 89, 256801 (2002).

[28] J. A. van Dam, Y. V. Nazarov, E. P. A. M. Bakkers, S. De Franceschi, and L. P. Kouwenhoven, Supercurrent reversal in quantum dots, Nature 442, 667 (2006).

[29] S. De Franceschi, L. Kouwenhoven, C. Schönenberger, and W. Wernsdorfer, Hybrid superconductor-quantum dot devices, Nat. Nanotechnol. 5, 703 (2010).

[30] J.-D. Pillet, C. H. L. Quay, P. Morfin, C. Bena, A. Levy Yeyati, and P. Joyez, Andreev bound states in supercurrentcarrying carbon nanotubes revealed, Nat. Phys. 6, 965 (2010).

[31] L. Bretheau, Ç. Ö. Girit, H. Pothier, D. Esteve, and C. Urbina, Exciting Andreev pairs in a superconducting atomic contact, Nature 499, 312 (2013).

[32] C. Janvier, L. Tosi, L. Bretheau, Ç. Ö. Girit, M. Stern, P. Bertet, P. Joyez, D. Vion, D. Esteve, M. F. Goffman, H. Pothier, and C. Urbina, Coherent manipulation of Andreev states in superconducting atomic contacts, Science 349, 1199 (2015).

[33] D. J. van Woerkom, A. Proutski, B. Van Heck, D. Bouman, J. I. Väyrynen, L. I. Glazman, P. Krogstrup, J. Nygård, L. P. Kouwenhoven, and A. Geresdi, Microwave spectroscopy of spinful Andreev bound states in ballistic semiconductor Josephson junctions, Nat. Phys. 13, 876 (2017).

[34] L. Tosi, C. Metzger, M. F. Goffman, C. Urbina, H. Pothier, S. Park, A. L. Yeyati, J. Nygård, and P. Krogstrup, SpinOrbit Splitting of Andreev States Revealed by Microwave Spectroscopy, Phys. Rev. X 9, 011010 (2019).

[35] L. Bretheau, Ç. Ö. Girit, C. Urbina, D. Esteve, and H. Pothier, Supercurrent Spectroscopy of Andreev States, Phys. Rev. X 3, 041034 (2013).

[36] A. Zazunov, V. S. Shumeiko, E. N. Bratus, J. Lantz, and G. Wendin, Andreev Level Qubit, Phys. Rev. Lett. 90, 087003 (2003).

[37] N. M. Chtchelkatchev and Y. V. Nazarov, Andreev Quantum Dots for Spin Manipulation, Phys. Rev. Lett. 90, 226806 (2003).

[38] S.-C. Zhang and J. Hu, A four-dimensional generalization of the quantum Hall effect, Science 294, 823 (2001).

[39] H. M. Price, O. Zilberberg, T. Ozawa, I. Carusotto, and N. Goldman, Four-Dimensional Quantum Hall Effect with Ultracold Atoms, Phys. Rev. Lett. 115, 195303 (2015).

[40] Y. E. Kraus, Z. Ringel, and O. Zilberberg, FourDimensional Quantum Hall Effect in a Two-Dimensional Quasicrystal, Phys. Rev. Lett. 111, 226401 (2013).

[41] M. Lohse, C. Schweizer, H. M. Price, O. Zilberberg, and I. Bloch, Exploring 4D quantum Hall physics with a 2D topological charge pump, Nature 553, 55 (2018).

[42] O. Zilberberg, S. Huang, J. Guglielmon, M. Wang, K. P. Chen, Y. E. Kraus, and M. C. Rechtsman, Photonic topological boundary pumping as a probe of $4 \mathrm{D}$ quantum Hall physics, Nature 553, 59 (2018).

[43] S. Sugawa, F. Salces-Carcoba, A. R. Perry, Y. Yue, and I. B. Spielman, Second Chern number of a quantumsimulated non-Abelian Yang monopole, Science 360, 1429 (2018).

[44] L. Lu, H. Gao, and Z. Wang, Topological one-way fiber of second Chern number, Nat. Commun. 9, 5384 (2018).

[45] C. Chan and X.-J. Liu, Non-Abelian Majorana Modes Protected by an Emergent Second Chern Number, Phys. Rev. Lett. 118, 207002 (2017).

[46] M. Kolodrubetz, Measuring the Second Chern Number from Nonadiabatic Effects, Phys. Rev. Lett. 117, 015301 (2016).

[47] M. Desjardins, L. C. Contamin, M. R. Delbecq, M. C. Dartiailh, L. E. Bruhat, T. Cubaynes, J. J. Viennot, F. Mallet, S. Rohart, A. Thiaville, A. Cottet, and T. Kontos, Synthetic spin-orbit interaction for Majorana devices, Nat. Mater. 18, 1060 (2019).

[48] E. Strambini, S. D’Ambrosio, F. Vischi, F. S. Bergeret, Y. V. Nazarov, and F. Giazotto, The $\omega$-SQUIPT as a tool to phase-engineer Josephson topological materials, Nat. Nanotechnol. 11, 1055 (2016).

[49] G. Yang, Z. Lyu, J. Wang, J. Ying, X. Zhang, J. Shen, G. Liu, J. Fan, Z. Ji, X. Jing, F. Qu, and L. Lu, Protected gap closing in Josephson trijunctions constructed on Bi2Te3, Phys. Rev. B 100, 180501 (2019).

[50] L. G. Herrmann, F. Portier, P. Roche, A. L. Yeyati, T. Kontos, and C. Strunk, Carbon Nanotubes as Cooper-Pair Beam Splitters, Phys. Rev. Lett. 104, 026801 (2010). 
[51] L. Hofstetter, S. Csonka, J. Nygård, and C. Schönenberger, Cooper pair splitter realized in a two-quantum-dot Y-junction, Nature 461, 960 (2009).

[52] A. I. Buzdin, Proximity effects in superconductorferromagnet heterostructures, Rev. Mod. Phys. 77, 935 (2005).

[53] M. V. Berry, Quantal phase factors accompanying adiabatic changes, Proc. R. Soc. A 392, 45 (1984).

[54] D. Xiao, M.-C. Chang, and Q. Niu, Berry phase effects on electronic properties, Rev. Mod. Phys. 82, 1959 (2010).

[55] N. Manton and P. Sutcliffe, Topological Solitons (Cambridge University Press, Cambridge, 2004).

[56] X.-L. Qi, T. L. Hughes, and S.-C. Zhang, Topological field theory of time-reversal invariant insulators, Phys. Rev. B 78, 195424 (2008).

[57] J. E. Avron, L. Sadun, J. Segert, and B. Simon, Topological Invariants in Fermi Systems with Time-Reversal Invariance, Phys. Rev. Lett. 61, 1329 (1988).

[58] Alexander, Monte-Carlo integration (https://www. mathworks.com/matlabcentral/fileexchange/53477-montecarlo-integration), MATLAB Central File Exchange. Retrieved May 16, 2020.

[59] M. Hays, V. Fatemi, K. Serniak, D. Bouman, S. Diamond, G. de Lange, P. Krogstrup, J. Nygård, A. Geresdi, and M. H. Devoret, Continuous monitoring of a trapped superconducting spin, Nat. Phys. 16, 1103 (2020).

[60] T. Ozawa and N. Goldman, Extracting the quantum metric tensor through periodic driving, Phys. Rev. B 97, 201117(R) (2018).

[61] D. J. Van Woerkom, A. Geresdi, and L. P. Kouwenhoven, One minute parity lifetime of a NbTiN Cooper-pair transistor, Nat. Phys. 11, 547 (2015).

[62] J. Petrides, H. M. Price, and O. Zilberberg, Six-dimensional quantum Hall effect and three-dimensional topological pumps, Phys. Rev. B 98, 125431 (2018).

[63] S. Sugawa, F. Salces-Carcoba, Y. Yue, A. Putra, and I. B. Spielman, Observation and characterization of a nonAbelian gauge field's Wilczek-Zee phase by the Wilson loop, arXiv:1910.13991 (2019).

[64] F. Leroux, K. Pandey, R. Rehbi, F. Chevy, C. Miniatura, B. Gremaud, and D. Wilkowski, Non-Abelian adiabatic geometric transformations in a cold strontium gas, Nat. Commun. 9, 3580 (2018).

[65] Y. Yang, C. Peng, D. Zhu, H. Buljan, J. D. Joannopoulos, B. Zhen, and M. Soljačić, Synthesis and observation of non-Abelian gauge fields in real space, Science 365, 1021 (2019).

[66] K. Toyoda, K. Uchida, A. Noguchi, S. Haze, and S.Urabe, Realization of holonomiw single-quit operations, Phys. Rev. A 87, 052307 (2013). 